\title{
Laser spectroscopic studies of state-dependent collisional quenching of the lifetimes of metastable antiprotonic helium atoms
}

\author{
M. Hori, H. A. Torii, R. S. Hayano, T. Ishikawa, F. E. Maas, ${ }^{*}$ and H. Tamura ${ }^{\dagger}$ \\ Department of Physics, University of Tokyo, 7-3-1 Hongo, Bunkyo-ku, Tokyo 113, Japan \\ B. Ketzer, F. J. Hartmann, R. Pohl, C. Maierl, M. Hasinoff, and T. von Egidy \\ Physik-Department, Technische Universität München, D-85747 Garching, Germany \\ M. Kumakura and N. Morita \\ Institute for Molecular Science, Myodaiji, Okazaki 444, Japan \\ I. Sugai \\ Institute for Nuclear Study, University of Tokyo, 3-2-1 Midori-cho, Tanashi, Tokyo 188, Japan \\ D. Horváth \\ KFKI Research Institute for Particle and Nuclear Physics, H-1525 Budapest, Hungary
}

E. Widmann, ${ }^{\ddagger}$ J. Eades, and T. Yamazaki ${ }^{\S}$

CERN, CH-1211 Geneva 23, Switzerland

(Received 12 August 1997; revised manuscript received 12 January 1998)

\begin{abstract}
A laser spectroscopic method was developed to directly observe the lifetimes of individual states in metastable antiprotonic helium atoms $\left(\bar{p}^{4} \mathrm{He}^{+}\right)$. Collisional effects with surrounding helium atoms were studied in cryogenic gas targets at pressures between 0.1 and 9.0 bars and at temperatures between 5.5 and $7.0 \mathrm{~K}$. The metastable state $(n, \ell)=(39,35)$ showed a lifetime of $1.5 \mu \mathrm{s}$, regardless of density, whereas the lifetime of the energetically lower-lying state $(37,34)$ was found to shorten from 1.3 to $0.1 \mu$ s with increasing density. The lifetimes of the states $(38,34)$ and $(36,33)$ were determined to be $10 \mathrm{~ns}$ and $5 \mathrm{~ns}$, respectively, showing the effects of the internal Auger process. When extrapolated to zero density, the measured lifetimes agreed with the results of calculations for the radiative and Auger widths. Using the fact that the state $(37,34)$ becomes short lived at high density, the resonance $(38,35) \rightarrow(37,34)$ at $529.62 \mathrm{~nm}$ was detected. [S1050-2947(98)01203-7]
\end{abstract}

PACS number(s): $36.10 . \mathrm{Gv}, 32.70 . \mathrm{Cs}, 34.90 .+\mathrm{q}$

\section{INTRODUCTION}

Some 3\% of all antiprotons stopped in dense helium are known to survive with a mean lifetime of 3-4 $\mu$ s [1-6]. This is due to the formation of metastable antiprotonic helium $\left(\bar{p} \mathrm{He}^{+}\right)$atoms $[7,8]$, which are neutral three-body Coulomb systems composed of a helium nucleus, an electron, and an antiproton that survive in spite of the high frequencies of collisions with ordinary helium atoms. Recently, laser spectroscopy techniques $[9,10]$ have enabled the direct measurement of the transition energies and lifetimes of individual states of this atom [10-19].

Antiprotonic atoms are formed when one of the electrons circling an ordinary atom is replaced by an antiproton, which

\footnotetext{
*Present address: Institut für Kernphysik, Universität Mainz, D55099 Mainz, Germany.

${ }^{\dagger}$ Present address: Physics Department, Tohoku University, Sendai 980-77, Japan.

${ }^{\ddagger}$ Present address: The Institute of Physical and Chemical Research (RIKEN), Wako, Saitama 351-01, Japan.

${ }^{\S}$ Present address: Japan Society for the Promotion of Science, 5-3-1 Kojimachi, Chiyoda-ku, Tokyo 102, Japan.
}

is captured into a highly excited state with a principal quantum number of $n \sim \sqrt{M^{*} / m_{e}}$ ( $M^{*}$ and $m_{e}$ are the reduced masses of the antiproton helium nucleus and electron-helium nucleus systems, respectively). These atoms typically annihilate within $1 \mathrm{ps}$, due to a variety of cascade mechanisms that quickly deexcite the antiproton into states with such small orbital angular momentum $(\mathscr{C}=0,1, \ldots)$ that their large overlap with the nucleus ensures rapid absorption. Thus antiprotonic hydrogen $(\bar{p} p)$ atoms formed in dense targets annihilate via collisional Stark processes with ordinary hydrogen atoms [20,21], while in antiprotonic atoms heavier than helium $(Z>2)$, the antiproton cascades down by internal Auger transitions with lifetimes of less than 1 ps [22]. In the latter process, part of the binding energy and angular momentum of the antiprotonic orbit is transferred to the remaining electron; this is ejected into the continuum, while the antiproton deexcites to an energetically lower-lying ionic state.

Antiprotonic helium atoms, however, appear to be unique, in that "circular" states with high orbital angular momentum and principal quantum number in the region where initial capture takes place $\left(n \approx \sqrt{M^{*} / m_{e}}=38.3\right.$ and $\left.\ell \approx n-1\right)$ have internal Auger lifetimes of microseconds or more 


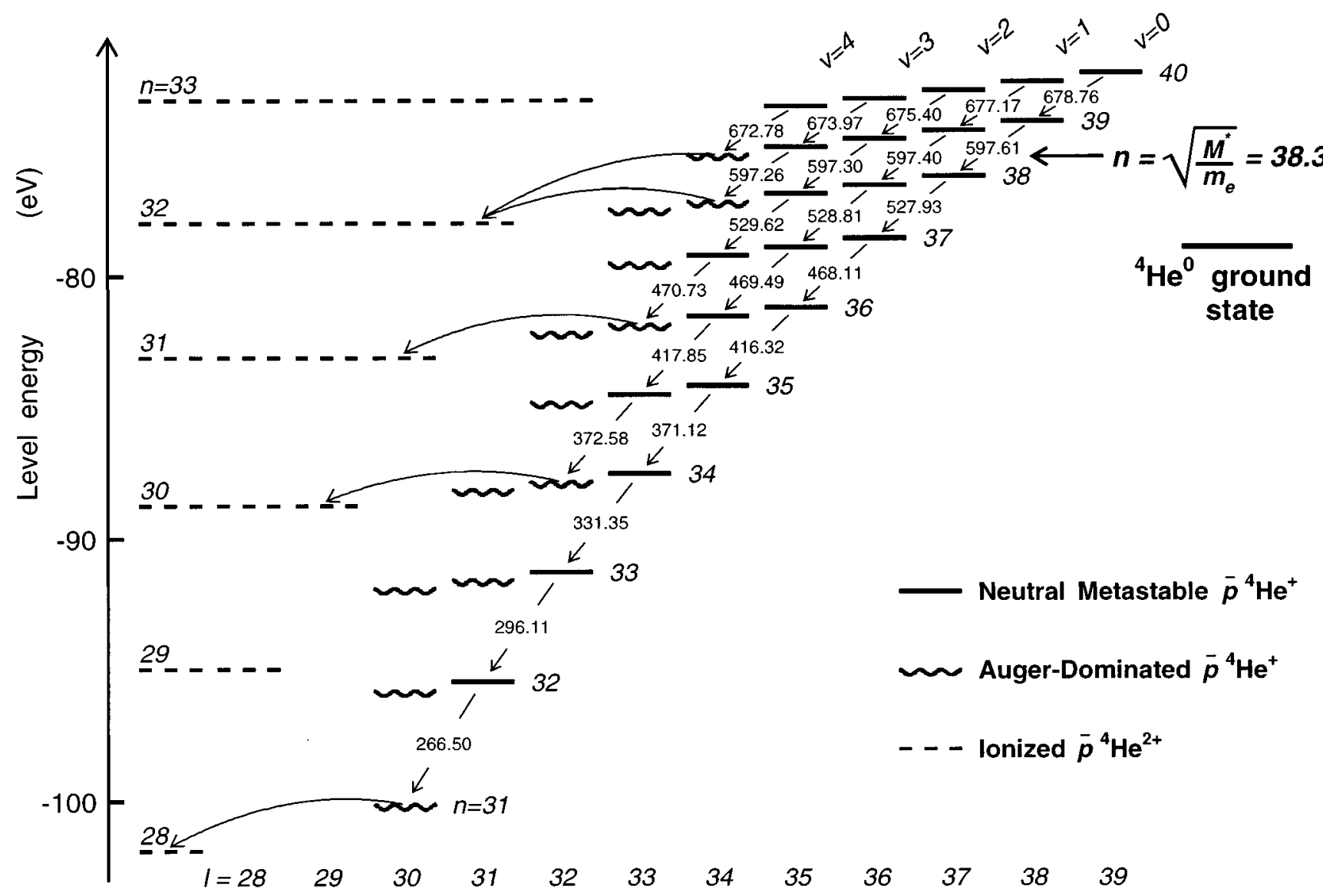

FIG. 1. Level structure of the $\bar{p}^{4} \mathrm{He}^{+}$atom. The solid lines indicate the radiation-dominated metastable levels with lifetimes of $1-2 \mu$ s, the wavy lines Auger-dominated short-lived states. The broken lines show the final $\bar{p} \mathrm{He}^{2+}$ ionic states formed after Auger electron emission. The curved arrows indicate Auger transitions with minimum $|\Delta \ell|$. On the left-hand scale the theoretical absolute energy of each state $(n, \ell)$ is plotted relative to the three-body-breakup threshold [33]. The calculated resonance wavelengths of radiative transitions following the constant- $v$ propensity rule are given in nanometers.

$[7,8,23]$. This is because the energy spacings of these antiprotonic orbitals $\left(E_{n}-E_{n-1} \sim 2 \mathrm{eV}\right.$; see Fig. 1) are small compared to the electronic ionization energy $(25 \mathrm{eV})$; Auger transitions to energetically lower-lying $\bar{p} \mathrm{He}^{2+}$ ionic states then require a large jump in both the $n$ value and the $\ell$ value of the antiprotonic orbital (e.g., $|\Delta n| \approx 6,|\Delta \ell| \geqslant 4$ ). Calculations show that the transition lifetime increases by three orders of magnitude as the multipolarity $L=|\Delta \mathscr{\ell}|$, or the angular momentum carried off by the emitted electron, increases by one unit $[9,24]$ (see Fig. 2). Hence the $\bar{p} \mathrm{He}^{+}$ states that are connected to a $\bar{p} \mathrm{He}^{2+}$ ionic state by angular momentum jump of $|\Delta \mathscr{\ell}| \geqslant 4$ have Auger lifetimes longer than $10 \mu \mathrm{s}$, while adjoining states from which $L \leqslant 3$ Auger transitions are possible have lifetimes of nanoseconds or less.

We use the term "metastable" for states with microsecond (or longer) Auger lifetimes. These states have the time to deexcite radiatively by dipole transitions, the lifetimes of which are 1-2 $\mu \mathrm{s}$ according to calculation [25-27]. The $\bar{p} \mathrm{He}^{+}$states in the $n \approx 38$ initial capture region are thus divided into two distinct regimes: the radiation-dominated metastable zone with large $\ell$ (indicated in Figs. 1 and 2 by solid lines) and the Auger-dominated short-lived zone with small $\ell$ (indicated by wavy lines). About $\sim 3 \%$ of the antiprotons stopped in dense gas or liquid helium are captured into the metastable levels [1-4] and cascade through the metastable states via successive $\tau=1-2 \mu$ s radiative transitions, these being primarily of the type $\Delta n=\Delta \ell=-1$ in the high- $\ell$ region $[25,26]$. This " propensity rule" conserves the radial node number or, equivalently, the vibrational quantum number $v=n-\mathscr{C}-1$, so that metastable states can be grouped into the "cascade chains"' $v=1,2,3, \ldots$ (see Fig. 1). After the antiproton reaches an Auger-dominated state, the atom proceeds to a $\bar{p} \mathrm{He}^{2+}$ ion within nanoseconds. The energy levels of this ion are now highly degenerate, so that Stark mixing results in antiproton annihilation through nuclear absorption within a picosecond $[28,29]$ as in the case of other antiprotonic atoms.

Laser-spectroscopy experiments have now confirmed both the existence of this metastable $\bar{p} \mathrm{He}^{+}$atom and the above description of its life history. A pulsed laser was used to induce transitions between metastable and Auger-dominated states, effectively forcing an immediate annihilation of otherwise long-lived antiprotons and thereby producing a sharp spike in the delayed annihilation time spectrum of the $\bar{p} \mathrm{He}^{+}$ atom [9]. One resonance detected at $597.26 \mathrm{~nm}$ (see Fig. 2) was ascribed to the transition $(n, \mathscr{C})=(39,35) \rightarrow(38,34)$ located at the end of the metastable cascade chain $v=3$ [10]. Another transition detected at $470.72 \mathrm{~nm}$ was ascribed to 


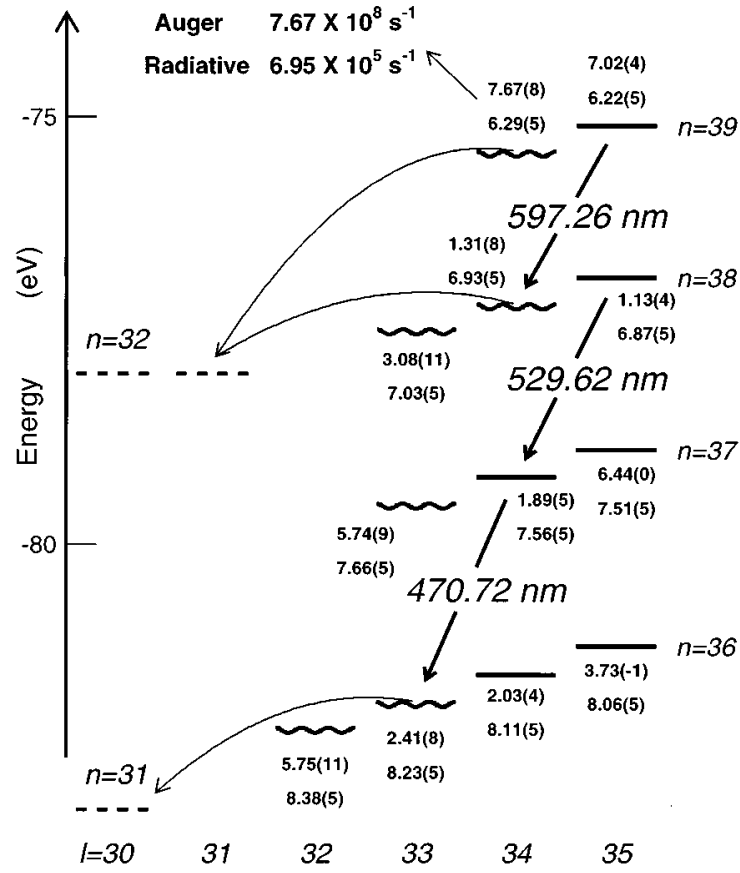

FIG. 2. Portion of the level structure of the $\bar{p}^{4} \mathrm{He}^{+}$atom, showing the positions of the experimentally observed 597.26-nm, 470.72-nm, and 529.62-nm resonances. The theoretical values for Auger [34] and radiative transition [27] rates of each state $(n, \ell)$ are indicated.

$(37,34) \rightarrow(36,33)$, where the $v=2$ cascade terminates [12].

The laser-spectroscopic method offered a way to map the state lifetimes in these two cascades, when supplemented by a simplified model of the cascade as described later [11]. In the $v=3$ cascade that was studied using helium gas targets at a pressure of $0.7-1$ bar and a temperature of 5-10 K, the states $(39,35)$ and $(40,36)$ showed lifetimes of $1.4 \mu$ s and $2.0 \mu \mathrm{s}$, respectively, in agreement with theoretical estimations [11]. However, measurements of the $v=2$ cascade done at 0.6 bar and $5.5 \mathrm{~K}$ indicated that the state $(37,34)$ had a lifetime of $0.8 \mu \mathrm{s}, 40 \%$ shorter than the theoretical radiative lifetime of $1.4 \mu \mathrm{s}$ [12]. It was speculated that the shorter lifetime was due to contributions from internal Auger process or from collisions with ordinary helium atoms.

Now it is interesting that the metastability of such states is retained in dense gas, liquid, and solid phases, despite the frequent collisions with the surrounding helium atoms. Contrary to the case of $\bar{p} p$ atoms, the continued presence of the electronic cloud in the $\bar{p} \mathrm{He}^{+}$atom causes the antiprotonic $\ell$ sublevels for a given $n$ to be far from degenerate, the level energies increasing with $\ell$ in steps of $\sim 0.3 \mathrm{eV}$ (see Fig. 1). The atom is thus quite insensitive to the collisional Stark effects that normally bring the antiproton to the $S, P$, and $D$ states at high $n$, from which annihilation occurs. Unfortunately, no quantitative theoretical understanding has yet been reached on this reduced sensitivity of the $\bar{p} \mathrm{He}^{+}$system to collisions with surrounding helium atoms.

Experiments have shown, however, that the overall lifetime of $\bar{p} \mathrm{He}^{+}$atoms does have a clear dependence on the density of the helium in which the atoms are formed [2-4]. In liquid helium targets with densities of $2 \times 10^{22} \mathrm{~cm}^{-3}$, the

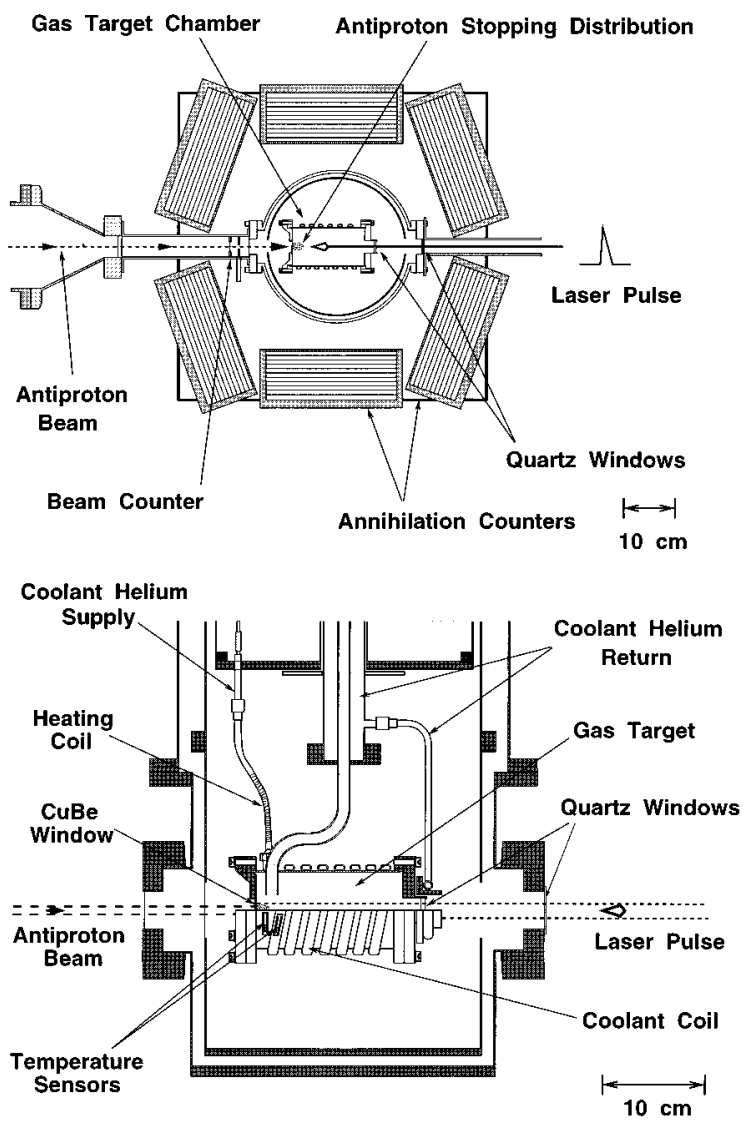

FIG. 3. Schematic layout of the laser spectroscopy experiment to measure individual state lifetimes of $\bar{p} \mathrm{He}^{+}$atoms (top). Antiprotons are stopped in dense helium with pressures from 0.1 to 9.0 bars and temperatures from 5.5 to $7.0 \mathrm{~K}$. Metastable $\bar{p} \mathrm{He}^{+}$atoms are irradiated by a laser pulse tuned to transition wavelengths 597.26 $\mathrm{nm}, 470.72 \mathrm{~nm}$, and $529.62 \mathrm{~nm}$, respectively. The lifetime of each atom is measured as the time elapsed from antiproton incidence (supplied by beam counters) to annihilation (supplied by shower counters). Side view of the cryogenic gas target chamber (bottom).

delayed annihilation time spectrum had a $\tau_{1}=0.2 \mu$ s shortlived component that accounted for $20-30 \%$ of the metastable $\bar{p} \mathrm{He}^{+}$atoms and a $\tau_{2}=2.5 \mu$ s long-lived component that contained the rest. In gas targets with densities of 2 $\times 10^{20} \mathrm{~cm}^{-3}$ (corresponding to target conditions of 1 bar and $30 \mathrm{~K}$ ), the short-lived component disappeared and the average lifetime was prolonged to $4 \mu \mathrm{s}$. However, to our knowledge no spectroscopic study existed prior to those described here on the effect of density on the lifetimes of individual states.

The present work reports systematic measurements of three resonance transitions at $597.26 \mathrm{~nm}, 470.72 \mathrm{~nm}$, and $529.62 \mathrm{~nm}$ to determine the lifetimes of four $\bar{p} \mathrm{He}^{+}$states and their dependence on the density of the surrounding helium. A laser spectroscopic method was developed to directly determine the lifetimes of individual states, and the measured lifetimes were compared with the results of recent calculations on the Auger [24,30-35] and radiative [25$27,30,33]$ widths. Finally, we show that some specific states become short lived in high-density helium, while others remain unaffected. 


\section{EXPERIMENTAL METHOD}

Our measurements are an extension of previous work $[3,10,19]$, the layout of which is shown in Fig. 3 (upper half). Antiprotons of 200-MeV/c momentum with a spread of $0.1 \%$ were extracted from the low-energy antiproton ring at CERN and stopped in a cryogenic helium gas target at rates of $10^{4} \mathrm{~s}^{-1}$, thus forming metastable $\bar{p} \mathrm{He}^{+}$atoms. The arrival of antiprotons was detected by a $0.5-\mathrm{mm}$-thick plastic scintillation beam counter, which was placed in the beam upstream of the target. The annihilation of each antiproton in the helium gas was identified with $(99.7 \pm 0.1) \%$ efficiency by seven shower counters surrounding the cryostat, which detected at least two of the five pions typically produced in the annihilation event. The annihilation time was thus measured relative to the time of passage of the antiprotons through the beam counter, one particle at a time. The $97 \%$ majority of the antiprotons that annihilated within $0.1 \mu \mathrm{s}$ after stopping in the target were rejected by a hardware veto circuit, so that the data-acquisition system was only required to handle the metastable atoms that annihilated later than 0.1 $\mu \mathrm{s}$. In this way, a background-free spectrum showing the decay time profile of metastable $\bar{p} \mathrm{He}^{+}$atoms between 0.1 and $15 \mu \mathrm{s}$ after atom formation could be obtained [3,5]. Henceforth, we refer to this as the delayed annihilation time spectrum of metastable $\bar{p} \mathrm{He}^{+}$atoms.

The metastable $\bar{p} \mathrm{He}^{+}$atoms were irradiated by a laser pulse fired into the target chamber, collinear with the antiproton beam but in the opposite direction. A dye laser pumped by a $\mathrm{XeCl}$ excimer laser was used to produce a laser pulse with a time width of $40 \mathrm{~ns}$, a diameter of $1 \mathrm{~cm}$, a power density of $1 \mathrm{~mJ} / \mathrm{cm}^{2}$ per pulse, and a bandwidth of $6-8 \mathrm{GHz}$. The laser was tuned by a calibrated pulse mode wavelength meter to the $\bar{p} \mathrm{He}^{+}$resonances at $597.26 \mathrm{~nm}, 470.72 \mathrm{~nm}$, and $529.62 \mathrm{~nm}$. Since these radiative transitions have a dipole amplitude of $0.2-0.3 \mathrm{D}$ [9], the power density of each pulse was well beyond the magnitude needed to saturate these resonances and attain the maximum resonance intensity.

The laser pulse reached the atom at a time 1.1-1.3 $\mu \mathrm{s}$ after formation or later. Irradiation at earlier times could enhance the resonance intensity, but this was the minimum delay achievable by the current method of identifying the randomly occurring metastable events and then triggering the excimer laser, which has an ignition delay of $\sim 0.8 \mu \mathrm{s}$. The ignition timing of each pulse was measured by a $p-i-n$ photodiode and used to correct for distortions in the resonance spike caused by the firing jitter of the laser, which was about \pm 20 ns.

In this way, two types of time spectra containing $10^{6}-10^{8}$ events were taken simultaneously, one with a single laser tuned to the resonance center, the other without laser irradiation. The measurements were repeated for the 597.26-nm and 470.72-nm resonances, for helium gas targets with an impurity content of less than $1 \mathrm{ppm}$, at pressures between 0.1 and 9.0 bars and temperatures between 5.5 and 7.0 K. High densities were reached with supercritical phase helium, which was preferable for many practical reasons to the use of a liquid phase target. The resonance wavelength was found to redshift as the density of the helium gas target was increased (as described elsewhere), so the laser wavelength was corrected for this shift at each target condition.

The cryogenic target chamber (see Fig. 3, lower half) was a stainless-steel cylinder $70 \mathrm{~mm}$ in diameter and $150 \mathrm{~mm}$ in length, into which the antiprotons entered through a copperberyllium window with a thickness of $0.5 \mathrm{~mm}$. The inside surface of this window was coated with aluminum to achieve $\sim 80 \%$ optical reflectivity and reduce the cryogenic heat load from the laser beam. For admitting the laser beam into the target gas a 20-mm-diam, 4-mm-thick quartz window was affixed to the opposite end of the chamber and sealed to withstand 10 bars of overpressure. A rectangular copper pipe was wound and brazed on the outside surface of the container and cold helium gas was circulated through this pipe, cooling the container by heat conduction. The target temperature was controlled by regulating this flow and by a heating coil around the pipe. Thermal diode sensors were installed on the copper coil and container surface, calibrated against the gas temperature near the antiproton stopping distribution with an accuracy of $\pm 0.1 \mathrm{~K}$. Absolute transducers (produced by MKS Instruments) measured the pressure in the external filling system at pressure equilibrium with the cryogenic target chamber with a precision of $\pm 5 \mathrm{mb}$ for targets below 1 bar, and $\pm 50 \mathrm{mb}$ for targets above 1 bar. The pressure and temperature were converted into atomic density [36,37] using the EPT-76 temperature scale. The calculated density may have differed from the local density around the atom during spectroscopic measurements since the $20 \%$ portion of the laser energy absorbed by the copperberyllium window became an $\sim 10-\mathrm{mW}$ heat source inside the cryogenic helium. Periodic changes in trigger rate and power deterioration of the laser dye and excimer gas also caused slow fluctuations of the target temperature. These effects have been included in the target-density error.

According to simulations [38] based on actual measurements of the characteristics of the antiproton beam [39], the particles that penetrated through the copper-beryllium window into the helium gas had a kinetic energy of 1-2 MeV with a spread of $0.2-0.3 \mathrm{MeV}$, a diameter of $8-10 \mathrm{~mm}$, and an emittance larger than $500 \pi \mathrm{mm}$ mrad due to multiple scattering in the various windows and counters upstream of the target. Therefore, the antiprotons had to be stopped in the helium gas as close to the window as possible, so that all the $\bar{p} \mathrm{He}^{+}$atoms would be in the 1-cm-diam collinear laser beam. In targets of lower density, polyamide degrader foils were added in the beam upstream of the target chamber to compensate for the smaller stopping power, although this caused a greater portion of the antiprotons to annihilate in the entrance window before reaching the target gas. The lowest usable target density with the available $200-\mathrm{MeV} / c$ beam was $10^{20} \mathrm{~cm}^{-3}$ (corresponding to helium at 0.1 bar and 6.3 K) for which simulations showed that $90 \%$ of the incoming antiprotons annihilated in the window.

\section{ANALYSIS AND RESULTS}

\section{A. Depletion-recovery spectrum}

Irradiation by an on-resonance laser pulse creates a sharp spike on the $\bar{p} \mathrm{He}^{+}$delayed annihilation time spectrum, which decays with the lifetime of the resonance daughter state. The spectrum then recovers with the lifetime of the 


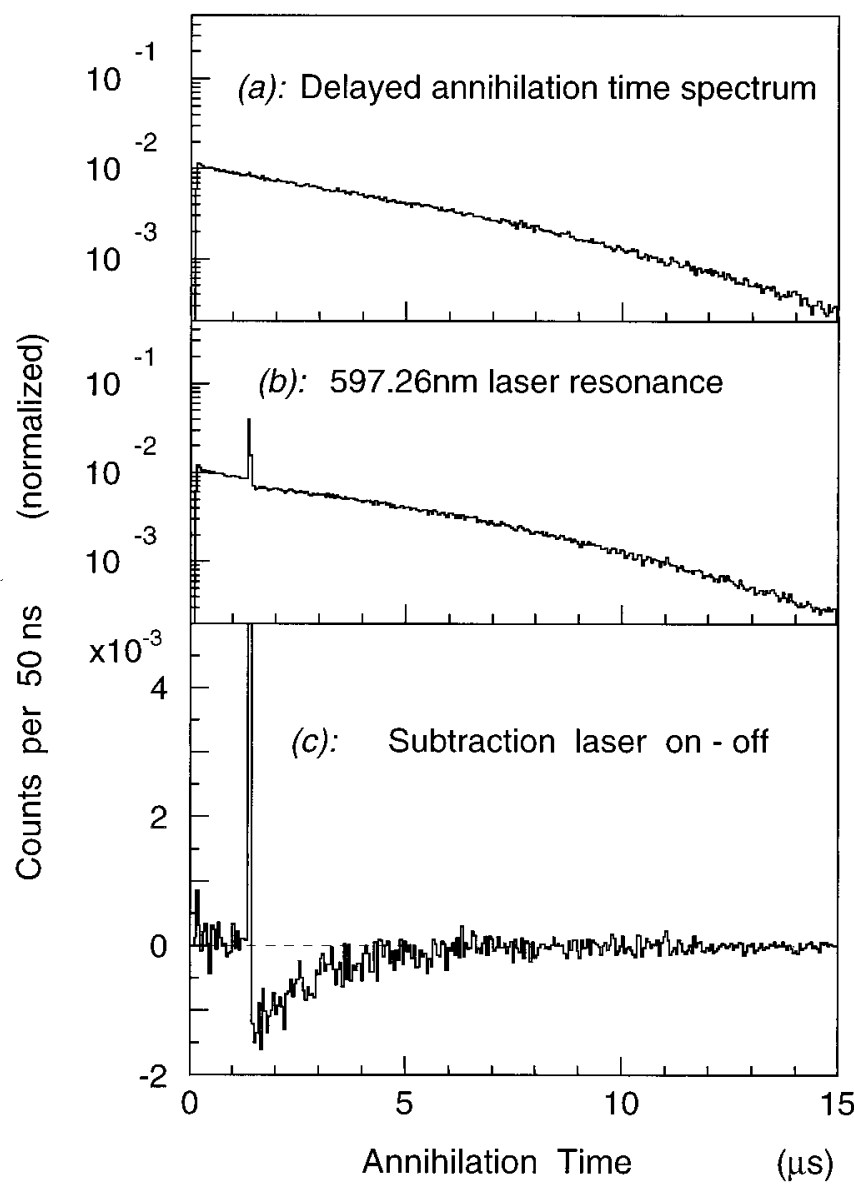

FIG. 4. (a) $\bar{p} \mathrm{He}^{+}$delayed annihilation time spectrum taken with ${ }^{4} \mathrm{He}$ gas target of 0.55 bar and $6.3 \mathrm{~K}$. (b) 579.26-nm resonance induced at $t=1.35 \mu \mathrm{s}$ on the time spectrum. (c) Depletion-recovery spectrum, the difference between the two normalized spectra (a) and (b).

parent state. These characteristics are valid regardless of the various channels that feed antiprotons into the two levels, so that this depletion-recovery method constitutes a sensitive and unambiguous way of determining the state lifetimes of $\bar{p} \mathrm{He}^{+}$atoms as can be seen from Fig. 4.

In Fig. 4(a) a time spectrum taken with a 0.55-bar and $6.3-\mathrm{K}$ target is plotted in a semilogarithmic scale, showing $\bar{p} \mathrm{He}^{+}$atoms decaying with a mean lifetime of $\sim 3 \mu \mathrm{s}$. The convex downward-curving profile is caused by antiprotons cascading through several metastable levels with microsecond lifetimes before annihilation. In Fig. 4(b) a resonance spike is produced at $t=1.35 \mu \mathrm{s}$, using a laser pulse tuned to the transition $(n, \mathscr{\ell})=(39,35) \rightarrow(38,34)$ at a wavelength of $597.26 \mathrm{~nm}$. The slight step-down discontinuity immediately afterward is caused by the loss of spontaneous annihilation events following the forced annihilation at the laser spike. The time spectrum in Fig. 4(c) is the difference between the two normalized spectra taken with and without the 597.26-nm irradiation. This subtraction effectively isolates the effect of the laser beam on the $\bar{p} \mathrm{He}^{+}$delayed annihilation time spectrum. The spectrum can be separated into three parts: (i) the time region $0.10-1.35 \mu \mathrm{s}$ preceding the resonance spike, where the spectrum is zero, (ii) the resonance spike itself (the next $\sim 40 \mathrm{~ns}$ ), and (iii) the depletion-

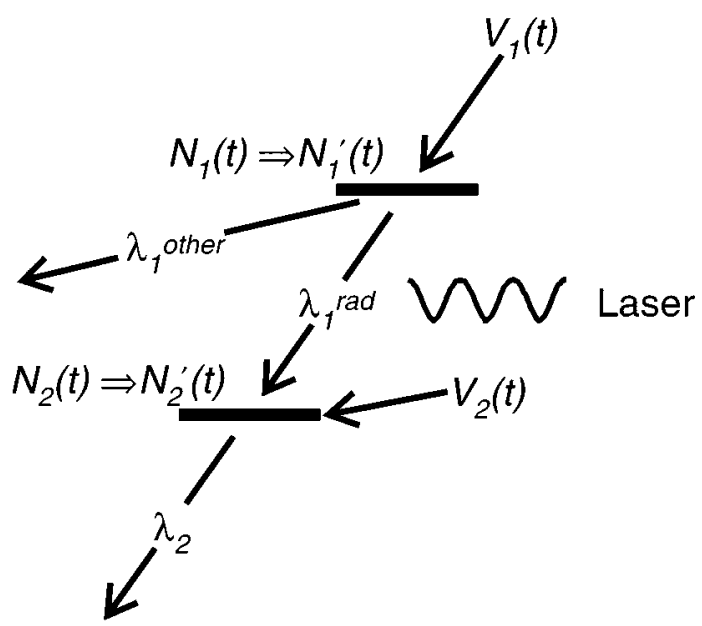

FIG. 5. Two-level laser resonance model. $V_{1}(t)$ and $V_{2}(t)$ represent feeding from surrounding states. The laser-induced resonance modifies the populations of the parent and daughter states, from $N_{1}(t)$ and $N_{2}(t)$ to $N_{1}^{\prime}(t)$ and $N_{2}^{\prime}(t)$.

recovery region following the spike, in which the spectrum gradually returns to zero over a period of a few microseconds. This depletion-recovery structure is due to those $\bar{p} \mathrm{He}^{+}$ atoms that annihilated in the resonance spike and are therefore "missing" from the spectrum following the spike. We could confirm that the total number of missing counts in the dip region, integrated to the end of the spectrum, is equal to the number of counts under the spike within the statistical errors.

To understand this depletion-recovery profile, consider the relevant two-level decay scheme depicted in Fig. 5. The total decay rates of the parent and daughter states of the resonance are denoted by $\lambda_{1}$ and $\lambda_{2}$, respectively. Here $\lambda_{1}$ is the sum of the radiative rate to the daughter state $\left(\lambda_{1}^{\mathrm{rad}}\right)$ and the decay rate to levels other than the daughter state $\left(\lambda_{1}^{\text {other }}\right)$. Denoting the net accumulation from adjacent states to the parent state by $V_{1}$ and the side feeding to the daughter state by $V_{2}$, the population evolution of the parent $\left(N_{1}\right)$ and daughter $\left(N_{2}\right)$ states follows

$$
\begin{gathered}
d N_{1}(t)=-\lambda_{1} N_{1}(t) d t+V_{1}(t) d t \\
d N_{2}(t)=-\lambda_{2} N_{2}(t) d t+\lambda_{1}^{\mathrm{rad}} N_{1}(t) d t+V_{2}(t) d t .
\end{gathered}
$$

When the on-resonance laser pulse irradiates the atom, Rabi oscillations mix the populations of the two levels. The resulting time spectrum profile has a complex shape depending on the resonance conditions, namely, the transition dipole moment, the laser power, and collisional broadening effects.

After the laser pulse, however, the time evolution resumes the form implied by Eqs. (1a) and (1b) with modified initial populations. The difference between the population evolutions with $\left(N_{1}^{\prime}, N_{2}^{\prime}\right)$ and without $\left(N_{1}, N_{2}\right)$ the resonance spike obeys 


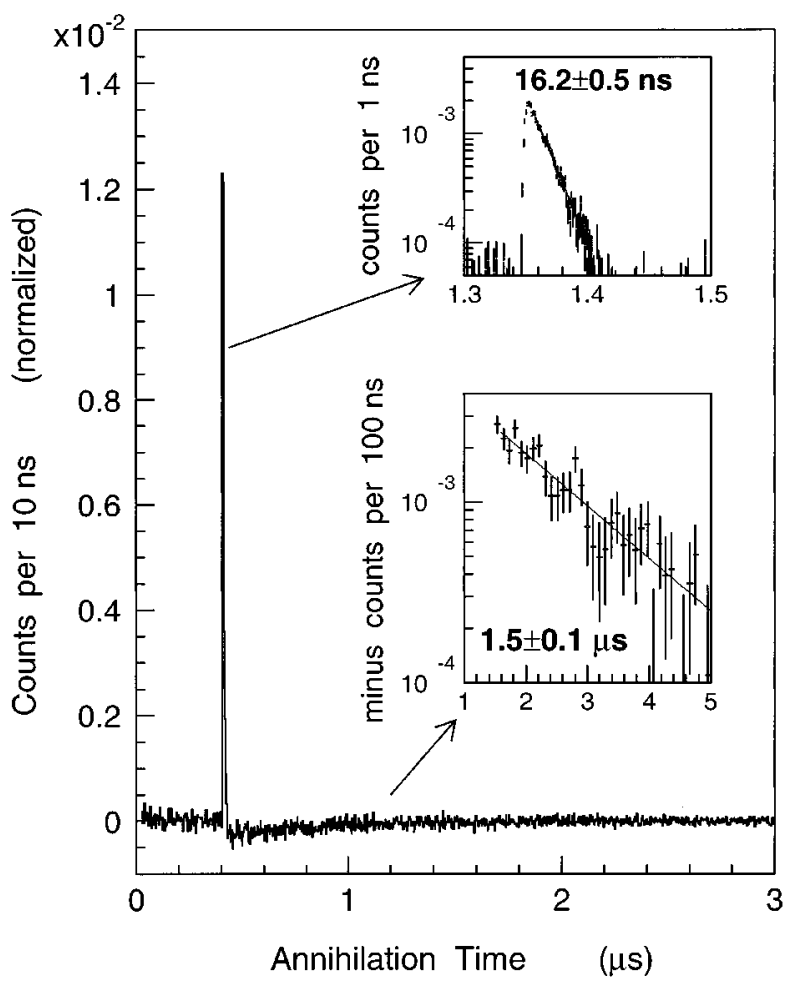

FIG. 6. Depletion-recovery spectrum of the 597.26-nm resonance at 0.55 bar and $6.3 \mathrm{~K}$. The lifetime of the resonance parent state can be derived from the recovery rate of this spectrum; in this case, the parent state $(n, \ell)=(39,35)$ has a lifetime of $1.5 \pm 0.1 \mu \mathrm{s}$ (lower inset with inverted scale).

$$
\begin{aligned}
d\left[N_{1}^{\prime}(t)-N_{1}(t)\right]= & -\lambda_{1}\left[N_{1}^{\prime}(t)-N_{1}(t)\right] d t, \\
d\left[N_{2}^{\prime}(t)-N_{2}(t)\right]= & -\lambda_{2}\left[N_{2}^{\prime}(t)-N_{2}(t)\right] d t \\
& +\lambda_{1}^{\mathrm{rad}}\left[N_{1}^{\prime}(t)-N_{1}(t)\right] d t
\end{aligned}
$$

Note that since the feeding from surrounding states $\left(V_{1}, V_{2}\right)$ is unaffected by the laser, the terms cancel by this subtraction. The solutions can then be expressed analytically as

$$
\begin{aligned}
N_{1}^{\prime}(t)-N_{1}(t) & =\left[N_{1}^{\prime}\left(t_{0}\right)-N_{1}\left(t_{0}\right)\right] e^{-\lambda_{1}\left(t-t_{0}\right)}, \\
N_{2}^{\prime}(t)-N_{2}(t)= & \frac{\lambda_{1}^{\mathrm{rad}}\left[N_{1}^{\prime}\left(t_{0}\right)-N_{1}\left(t_{0}\right)\right]}{\lambda_{2}-\lambda_{1}} e^{-\lambda_{1}\left(t-t_{0}\right)} \\
& +\left[N_{2}^{\prime}\left(t_{0}\right)-N_{2}\left(t_{0}\right)\right. \\
& \left.-\frac{\lambda_{1}^{\mathrm{rad}}\left[N_{1}^{\prime}\left(t_{0}\right)-N_{1}\left(t_{0}\right)\right]}{\lambda_{2}-\lambda_{1}}\right] e^{-\lambda_{2}\left(t-t_{0}\right)},
\end{aligned}
$$

where $t_{0}$ is the time at the end of the laser pulse.

Before the arrival of the laser pulse, the metastable parent state contains a larger population than the Auger-dominated daughter state $\left[\lambda_{1} \ll \lambda_{2}, N_{1}\left(t_{0}\right)>N_{2}\left(t_{0}\right)\right]$. The laser pulse causes the parent population to decrease $\left[N_{1}^{\prime}\left(t_{0}\right)<N_{1}\left(t_{0}\right)\right]$, while the daughter population is increased $\left[N_{2}^{\prime}\left(t_{0}\right)>N_{2}\left(t_{0}\right)\right]$. The depletion-recovery time spectrum implied by Eqs. (3a) and $(3 b)$ therefore decays with the daughter lifetime $\left(1 / \lambda_{2}\right)$ and recovers with the parent lifetime $\left(1 / \lambda_{1}\right)$, so that the lifetimes of the two states can be directly derived from the shape of the time spectrum.

In Fig. 6 the depletion-recovery spectrum of the 597.26-nm resonance taken at a target pressure and temperature combination of 0.55 bar and $6.3 \mathrm{~K}$ is shown. The recovery rate of the time spectrum after the resonance spike indicates a lifetime of $1.5 \pm 0.1 \mu \mathrm{s}$ for the resonance parent state $(39,35)$. This lifetime agrees with the result of earlier experiments at similar target conditions using the " $t_{1}-t_{2}$ " method [11].

\section{B. Comparison to previous methods}

In previous experiments, the lifetimes and initial populations of the $v=2$ and 3 cascades were measured $[11,12]$ by the $t_{1}-t_{2}$ method. Each $\bar{p} \mathrm{He}^{+}$atom was irradiated with two successive laser pulses tuned to the same resonance at independently varying times after formation of the $\bar{p} \mathrm{He}^{+}$atom. The first pulse at time $t_{1}$ induced annihilation from the last metastable state of a constant $v$ cascade sequence, the number of such annihilations thus constituting a measure of the population of that state at $t_{1}$. Natural feeding from higher levels in the cascade then refilled the parent state. The number of annihilations induced by a second laser pulse at time $t_{2}$ then measured the recovery of the parent state population between $t_{1}$ and $t_{2}$, and the population evolution of the parent state was obtained. Since this evolution is determined by the cumulative summation of antiprotons cascading through multiple states, cascade models with two- and three-level sequences of $\Delta n=\Delta \ell=-1$ transitions were used to extract the lifetimes of individual states from these measurements. Of course, there are also contributions from radiative processes that change $v=n-l-1$ and furthermore internal Auger processes, but calculations for the case of single isolated atoms suggest that these contributions are all less than $10 \%$ $[9,25-27,30]$.

In the high-density regime, however, the cascade may no longer be purely radiative. The profile of the delayed annihilation time spectrum is known to change with density, indicating that collisional processes affect state lifetimes. To study density effects on lifetimes, the depletion-recovery method is preferable to the $t_{1}-t_{2}$ method since the decay rates of the resonance parent and daughter states appear directly in the observed time spectrum and its results do not depend on cascade models.

\section{Lifetimes of the radiation-dominated states $(n, \ell)=(39,35)$ and $(37,34)$}

The lifetimes of the metastable states $(n, \ell)=(39,35)$ and $(37,34)$, which are the parent states of the 597.26-nm and 470.72-nm resonances, respectively, were determined at various densities using the depletion-recovery method. The intensity of the $597.26 \mathrm{~nm}$ resonance spike measured at densities between $2 \times 10^{20} \mathrm{~cm}^{-3}$ (corresponding to a target condition of 0.2 bar and $6.8 \mathrm{~K})$ and $1.9 \times 10^{22} \mathrm{~cm}^{-3}(8.7$ bars and $5.8 \mathrm{~K}$ ) is relatively constant (Fig. 7, left), indicating that 

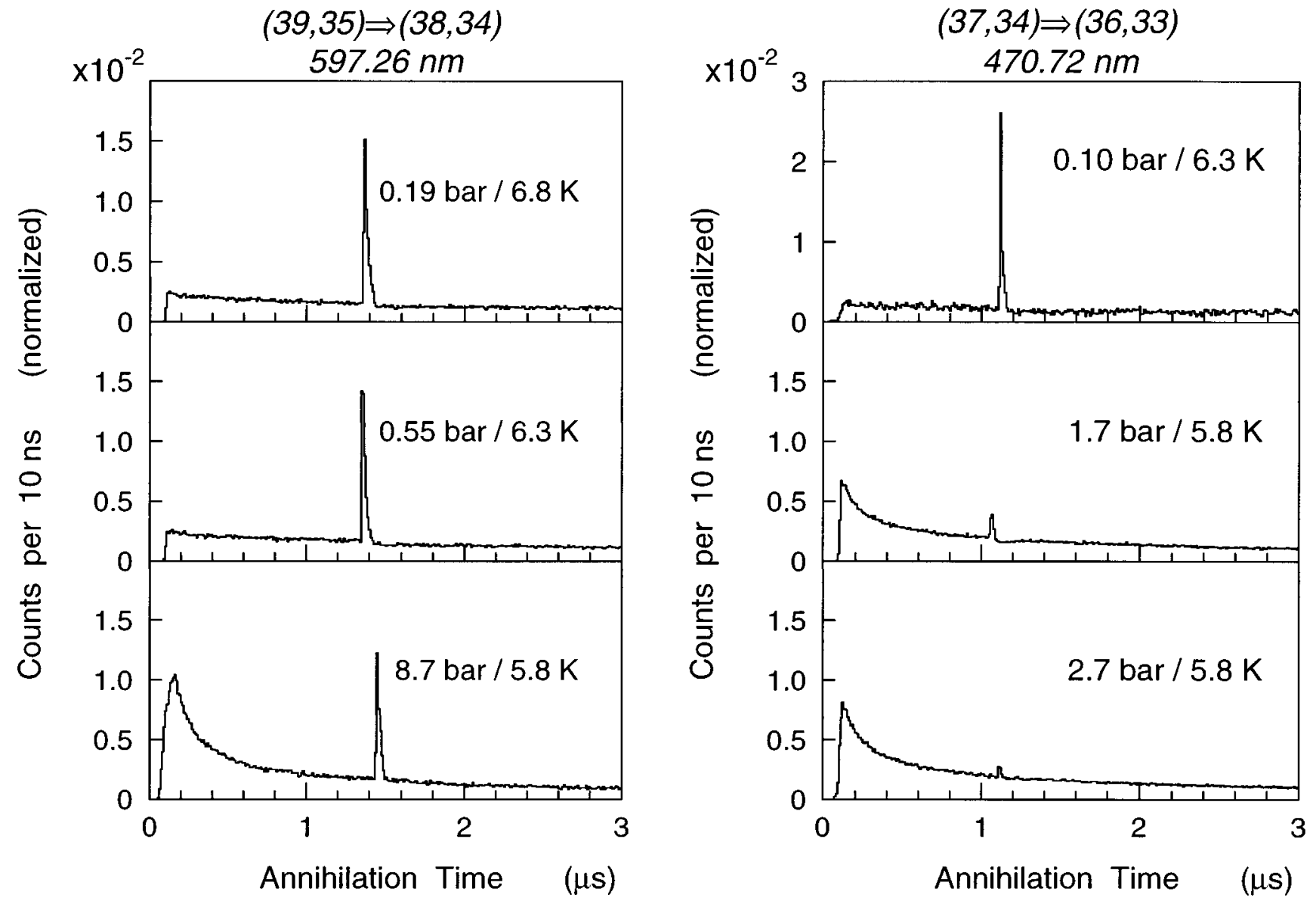

FIG. 7. Profiles of the two resonances $(n, \ell)=(39,35) \rightarrow(38,34)$ at $597.26 \mathrm{~nm}$ (left), and $(37,34) \rightarrow(36,33)$ at $470.72 \mathrm{~nm}($ right), measured at various densities. The 597.26-nm resonance intensity is independent of density, whereas that of the 470.72-nm resonance diminishes as the density increases.

the state lifetimes and initial populations of states in the $v=3$ cascade are unaffected by the increase of collision rates with ordinary helium atoms in this density range. The recovery rate of the time spectrum after the resonance depletion showed the lifetime of the resonance parent state $(39,35)$ was constant at $\sim 1.5 \mu$ s regardless of density (Fig. 8, left).

In contrast, a drastic density effect was observed for the 470.72-nm resonance (Fig. 7, right). In the spectrum taken at a target density of $\rho=1.2 \times 10^{20} \mathrm{~cm}^{-3}(0.1$ bar and $6.4 \mathrm{~K})$, the area under the resonance spike contained more than $4 \%$ of the total metastable $\bar{p} \mathrm{He}^{+}$fraction, but as the density was increased to $6 \times 10^{21} \mathrm{~cm}^{-3}(2.7$ bars and $5.8 \mathrm{~K})$, this diminished to about $0.2 \%$. The decrease of the spike intensity indicates that high densities reduced the lifetime of the resonance parent state $(37,34)$, resulting in a reduced population of the state when the laser pulse arrived at $t=1.1 \mu \mathrm{s}$. The depletion-recovery time spectrum (Fig. 8, right) shows that as the density increases from $\rho=1.2 \times 10^{20}$ to $3 \times 10^{21} \mathrm{~cm}^{-3}$, the state lifetime decreases from $\tau=1.2$ to $0.1 \mu \mathrm{s}$ and then levels off at higher densities.

\section{Lifetimes of the Auger-dominated states $(n, \ell)=(38,34)$ and $(36,33)$}

Next we determined the lifetimes of the Auger-dominated states $(n, \mathscr{\ell})=(38,34)$ and $(36,33)$, which are the daughter states of the 597.26-nm and 470.72-nm resonances, by measuring the decay rates of the resonance spikes. In the time spectrum of Fig. 6, the 597.26-nm resonance spike apparently decays with a lifetime of $\tau \sim 16 \mathrm{~ns}$, while in earlier work similar lifetimes have been noted for the 597.26-nm [10] and 470.72-nm [12] spikes. However, these are only the upper limits of the actual lifetime of the daughter states $[9,12]$ since during the 40 ns-long laser pulse, Rabi oscillations under dephasing collisions cause relaxation of the resonance spike profile and also the time structure of the laser pulse is convoluted into the spike, deforming its shape. When the laser pulse ends, however, the Rabi oscillations cease and the spike begins to decay purely with the lifetime of the resonance daughter state, until the population of the state is fully depleted.

The 597.26-nm resonance spike was measured at densities between $2 \times 10^{20}$ and $1.9 \times 10^{22} \mathrm{~cm}^{-3}$ (Fig. 9, upper left) and the lifetime of the spike after the end of the 40-ns laser pulse was obtained. The derived lifetimes of the state $(38,34)$ were distributed around $\tau=11 \pm 3$ ns (Fig. 9, lower left).

The 470.72-nm resonance spikes (Fig. 9, upper right) show a two-peak structure, which is a distortion caused by the time structure of the excimer laser pulse. The more pronounced effect of this structure on the 470.72-nm resonance spike than in the 597.26-nm spike is a consequence of the 

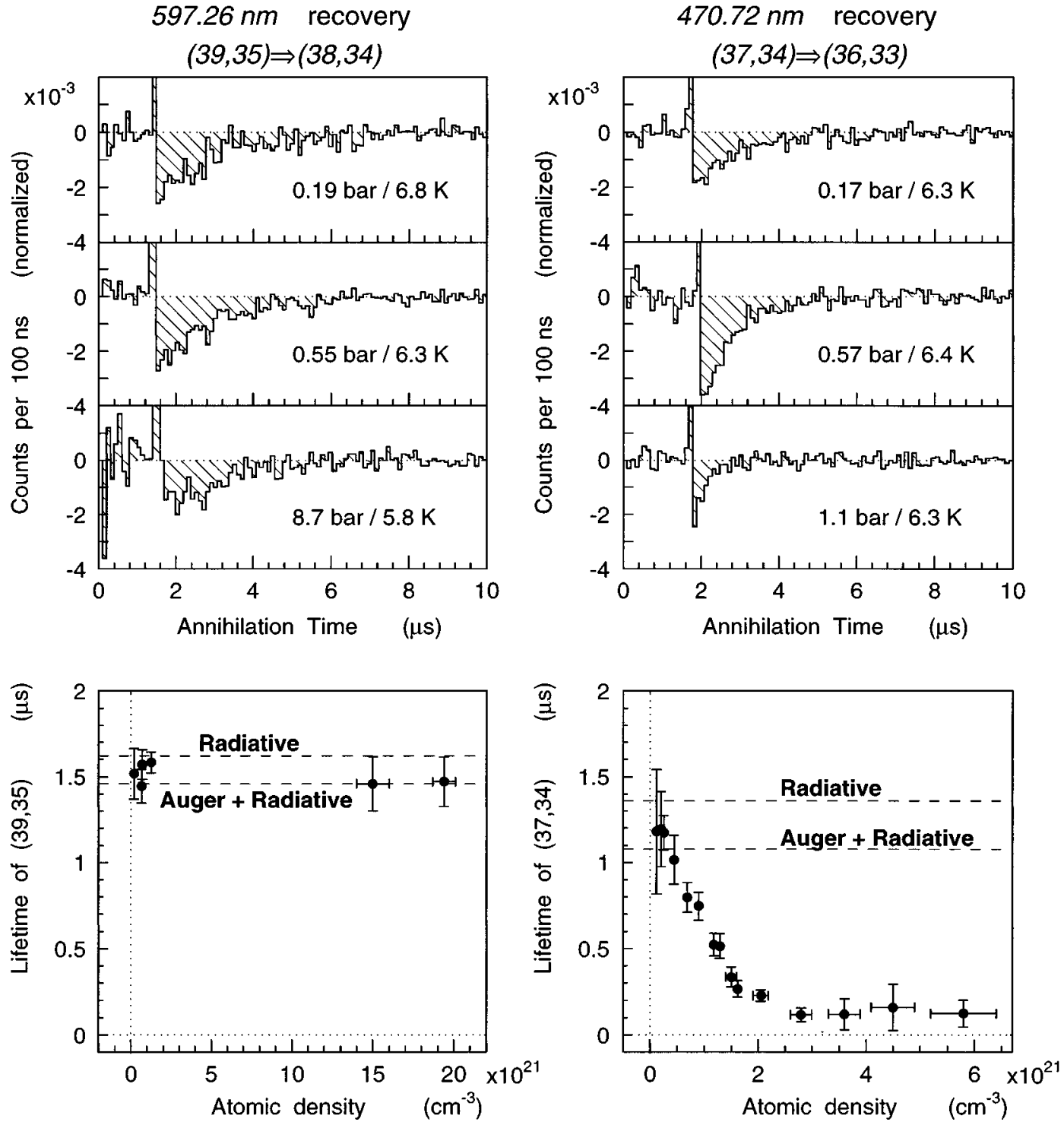

FIG. 8. Depletion-recovery spectra of the 597.26-nm (left) and 470.72-nm (right) resonances at various target densities. The lifetimes of the radiation-dominated parent states $(n, \ell)=(39,35)$ and $(37,34)$ are plotted as a function of density. The $(37,34)$ state becomes short lived with increased density, while the higher-lying $(39,35)$ remains unaffected. Theoretical radiative rates and the sum of radiative and Auger rates are also shown $[33,34]$.

shorter lifetime of the daughter state $(36,33)$ of the former resonance, the short lifetime causing the spike to decay almost completely within the $40 \mathrm{~ns}$ length of the laser pulse. Since the spike intensity decreases at high densities, we used comparably low-density targets between $10^{20}$ and $10^{21} \mathrm{~cm}^{-3}$. A lifetime of $\tau=5 \pm 2 \mathrm{~ns}$ was thus derived for the daughter state $(36,33)$.

\section{E. Detection of the 529.62-nm resonance}

The resonance transition $(n, \ell)=(38,35) \rightarrow(37,34)$ at $529.62 \mathrm{~nm}$ was observed using the phenomenon that the resonance daughter state becomes short lived at high density. Normally, at densities below $5 \times 10^{20} \mathrm{~cm}^{-3}$, detection of this transition as a single resonance is difficult because both parent and daughter states are metastable with $\sim 1-\mu$ s lifetimes and the laser pulse can only effect the net transfer of antiprotons between the two states if the population of these differs. Previously, a double-resonance method using two simultaneous laser pulses was employed to detect the transition [14].

To distinguish the laser-induced annihilation from the spontaneously decaying $\bar{p} \mathrm{He}^{+}$atoms comprising the continuous spectrum, (i) the lifetime of the resonance spike (and hence that of the daughter state) must be less than $0.5 \mu$ s and (ii) there must be a population difference between parent and daughter states before the arrival of the laser pulse. Favorable conditions to observe the resonance could be achieved with target densities of $1.3 \times 10^{21} \mathrm{~cm}^{-3}$ (corresponding to 1.0 bar and $6.5 \mathrm{~K}$ ) as the lifetime of the daughter state $(37,34)$ shortens to $0.5 \mu \mathrm{s}$ at this value (see Fig. 8 , right). As expected, when such a target density was used and the laser tuned to the resonance wavelength of $529.62 \mathrm{~nm}$ [14], a resonance spike was detected, which decayed with a lifetime of $0.5 \mu \mathrm{s}$ (Fig. 10). 
$(39,35) \Rightarrow(38,34)$
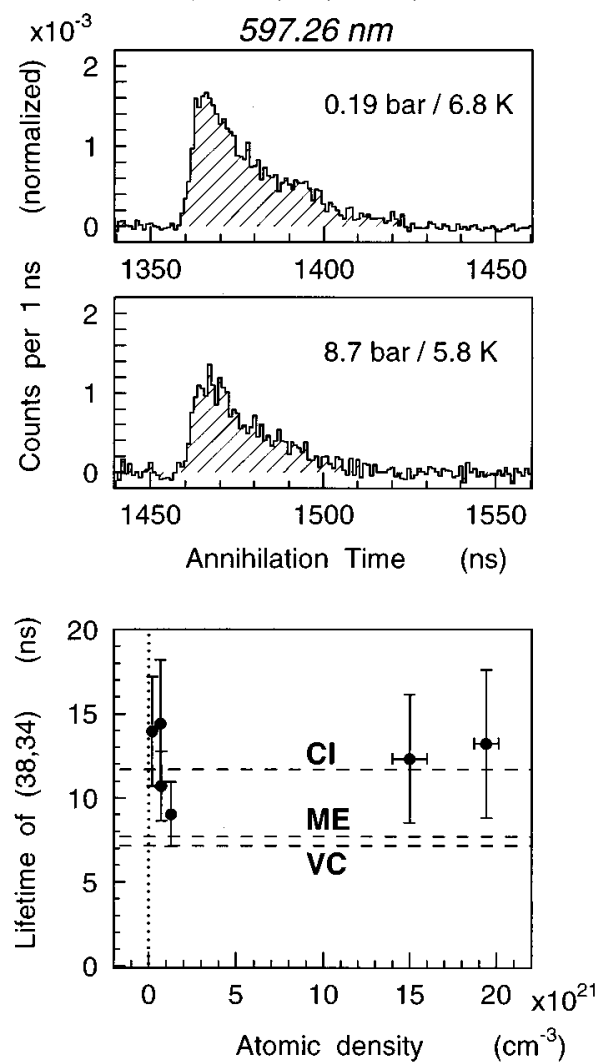

$(37,34) \Rightarrow(36,33)$

$\times 10^{-3} \quad 470.72 \mathrm{~nm}$
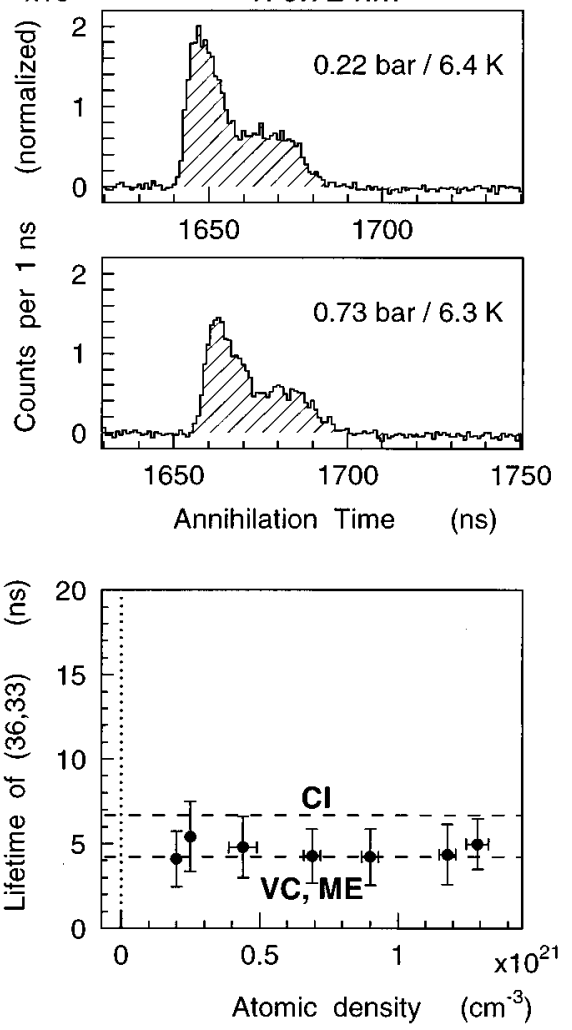

FIG. 9. Detail of the 597.26-nm (left) and 470.72-nm (right) resonance spikes at various densities. The derived lifetimes of the Auger-dominated states $(n, \ell)=(38,34)$ and $(36,33)$ are plotted as a function of density. The Auger rates derived from atomic configurationinteraction (CI [24]) and molecular variational (VC [30,31] and ME [34]) calculations are shown.

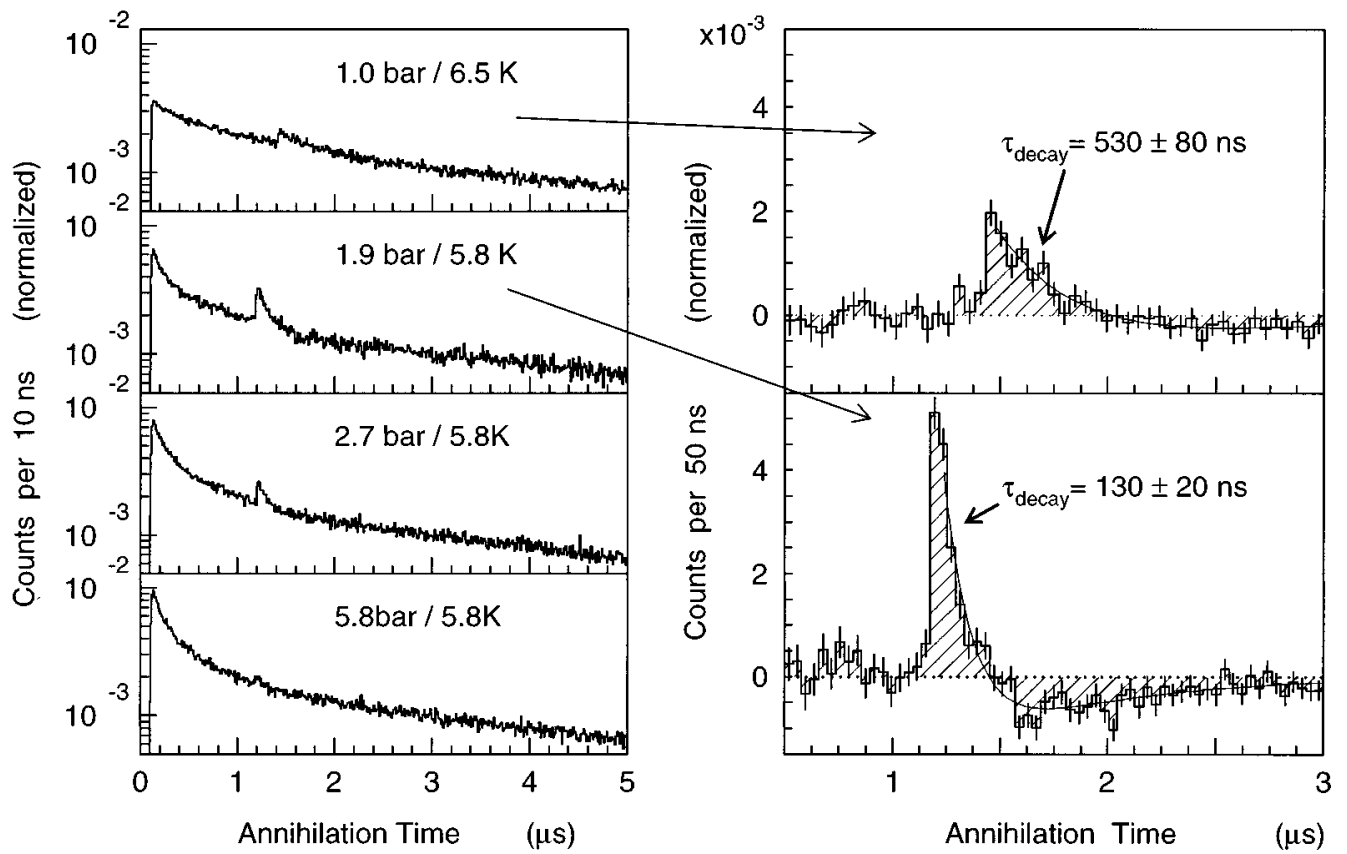

FIG. 10. Slowly decaying spike of the $(n, \ell)=(38,35) \rightarrow(37,34)$ resonance at $529.62 \mathrm{~nm}$, measured at four target densities $($ left $)$. The depletion-recovery spectrum of the resonance, showing the decay rates ( $\left.\tau_{\text {decay }}\right)$ of the spike (right). The effect of the lifetime shortening of the resonance daughter state $(37,34)$ can be seen. 
$(39,35)$

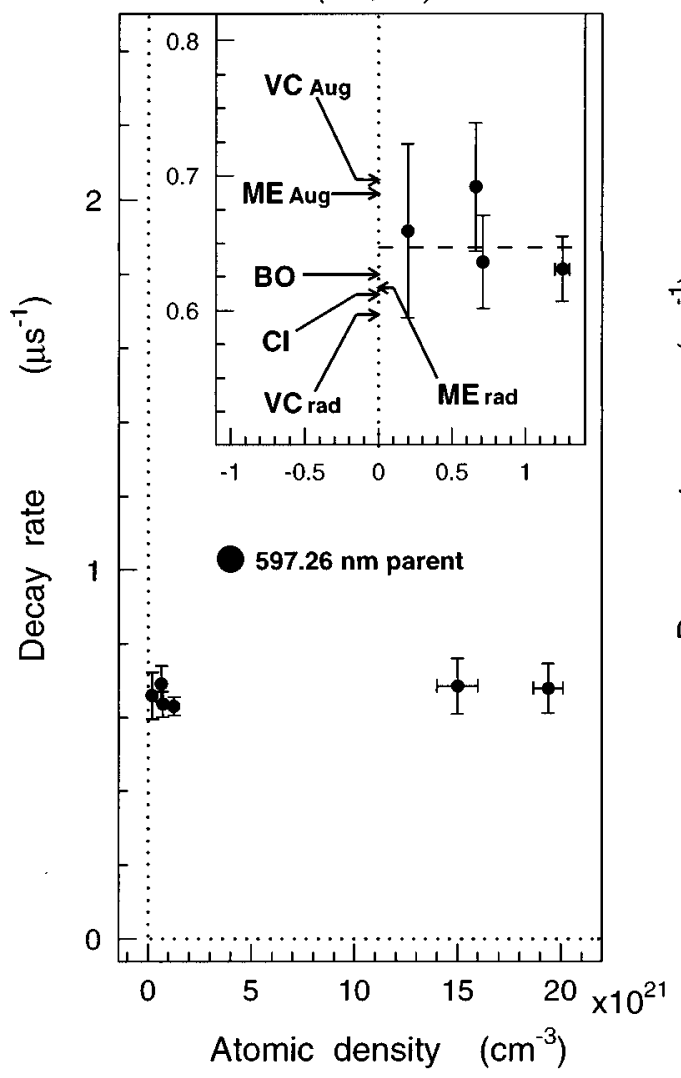

$(37,34)$

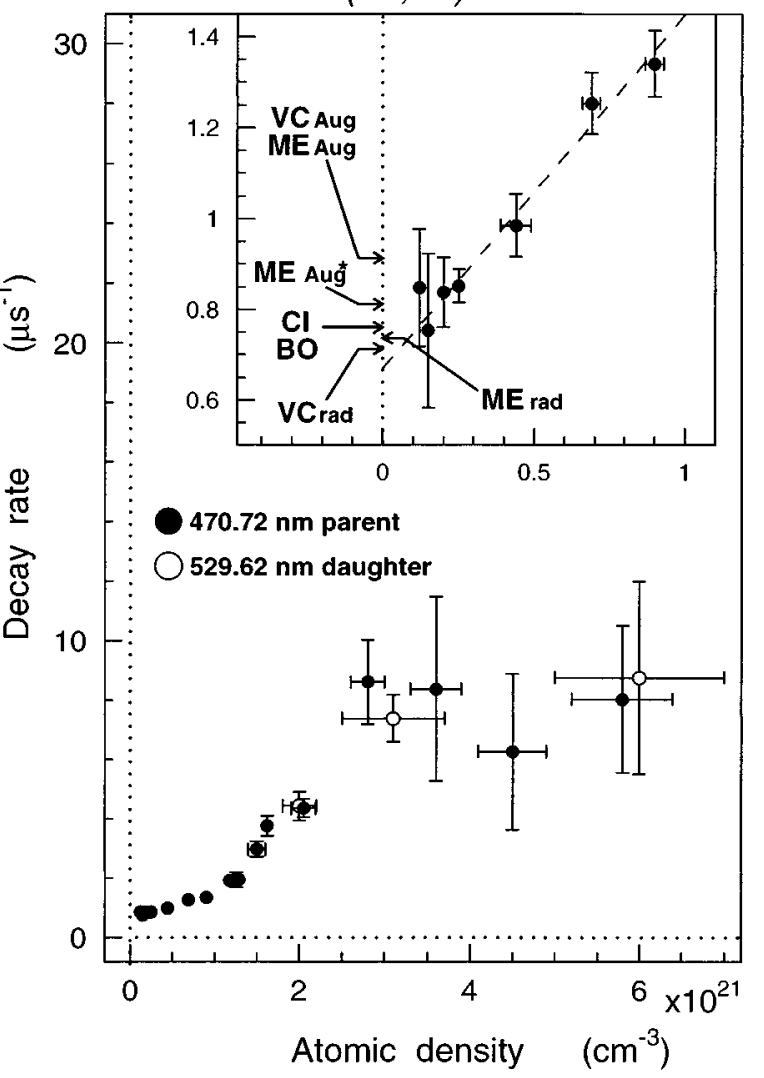

FIG. 11. The $\bar{p} \mathrm{He}^{+}$delayed annihilation time spectra at four densities (left) and the decay rates of the short-lived and long-lived components as a function of density (right). The rates are obtained from a double exponential fitted to the early-time region $(0.1-3.0 \mu s)$.

The depletion-recovery spectrum showed that as the target density was increased from $1.3 \times 10^{21}$ to $3 \times 10^{21} \mathrm{~cm}^{-3}$ $(1.9$ bars and $5.8 \mathrm{~K})$, the lifetime of the resonance daughter state $(37,34)$ shortened from 0.5 to $0.1 \mu$ s (which agrees with the results of the measurements using the 470.72-nm resonance), whereas the parent state retained a lifetime which was longer than $0.6 \mu \mathrm{s}$. In this region, the resonance intensifies with higher densities because the reduced lifetime of the daughter state ensures a larger difference between the populations of the two states at the arrival time of the laser pulse. At a density of $6 \times 10^{21} \mathrm{~cm}^{-3}(2.7$ bars and $5.8 \mathrm{~K})$, however, the resonance intensity decreased, and finally the spike nearly disappeared at a density of $1.6 \times 10^{22} \mathrm{~cm}^{-3}$ ( 5.8 bars and $5.8 \mathrm{~K}$ ). This behavior is related to the density dependence of the parent and daughter state populations, which will be described elsewhere.

\section{F. The short-lived component in the delayed annihilation time spectrum}

In Fig. 11 (left), the delayed annihilation time spectra measured at various densities between $1.2 \times 10^{20} \mathrm{~cm}^{-3}(0.1$ bar and $6.3 \mathrm{~K})$ and $1.9 \times 10^{22} \mathrm{~cm}^{-3}(8.7$ bars and $5.8 \mathrm{~K})$ are shown. Previous studies have demonstrated that cascade models with three or four components are needed to fit the entire profile of the time spectrum [1-5], but here we restrict our interest to the part of the spectrum immediately following $\bar{p} \mathrm{He}^{+}$atom formation, i.e., between $t=0.1$ and $3.0 \mu \mathrm{s}$, as this is the most sensitive area to changes in the density of the target gas. The time spectra were fitted with double exponentials for the time range $t=0.1-3.0 \mu \mathrm{s}$ and the time constants of the two components (one long lived, the other short lived) are plotted as a function of density (Fig. 11, right). As the target density was increased from $1.2 \times 10^{20}$ to $4 \times 10^{20} \mathrm{~cm}^{-3}(0.37$ bar and $6.3 \mathrm{~K})$, a short-lived component appeared at early times in the time spectrum, with a decay rate increasing sharply from 1 to $\sim 25 \mu \mathrm{s}^{-1}$. At $\rho=9 \times 10^{20}$ $\mathrm{cm}^{-3}(0.73$ bar and $6.3 \mathrm{~K})$, the component decayed completely within $0.1 \mu \mathrm{s}$ after antiproton arrival in the target gas and was therefore eliminated from the recorded time spectrum by the veto of prompt annihilation events mentioned earlier.

As the density was further increased from $9 \times 10^{20}$ to $3 \times 10^{21} \mathrm{~cm}^{-3}(1.7$ bars and $5.8 \mathrm{~K})$, a second short-lived component appeared in the time spectrum, the decay rate of which increased from 2 to $6 \mu \mathrm{s}^{-1}$ and then leveled off at higher densities, reaching $\lambda=7 \mu \mathrm{s}^{-1}$ at a liquid helium density of $\rho=1.9 \times 10^{22} \mathrm{~cm}^{-3}(8.7$ bars and $5.8 \mathrm{~K})$.

\section{DISCUSSION}

The observed decay rate of state $(n, \ell)$ at a target density $\rho$ may be written

$$
\lambda_{n, \ell}(\rho)=R_{n, \ell}+A_{n, \ell}+D_{n, \ell}(\rho),
$$

where the net radiative and internal Auger rates of state $(n, \ell)$ at zero density are 
TABLE I. Decay rates of the radiation-dominated states $(n, \ell)=(39,35)$ and $(37,34)$ and the Auger-dominated states $(38,34)$ and $(36,33)$ extrapolated to zero density. Theoretical radiative and internal Auger decay rates are included.

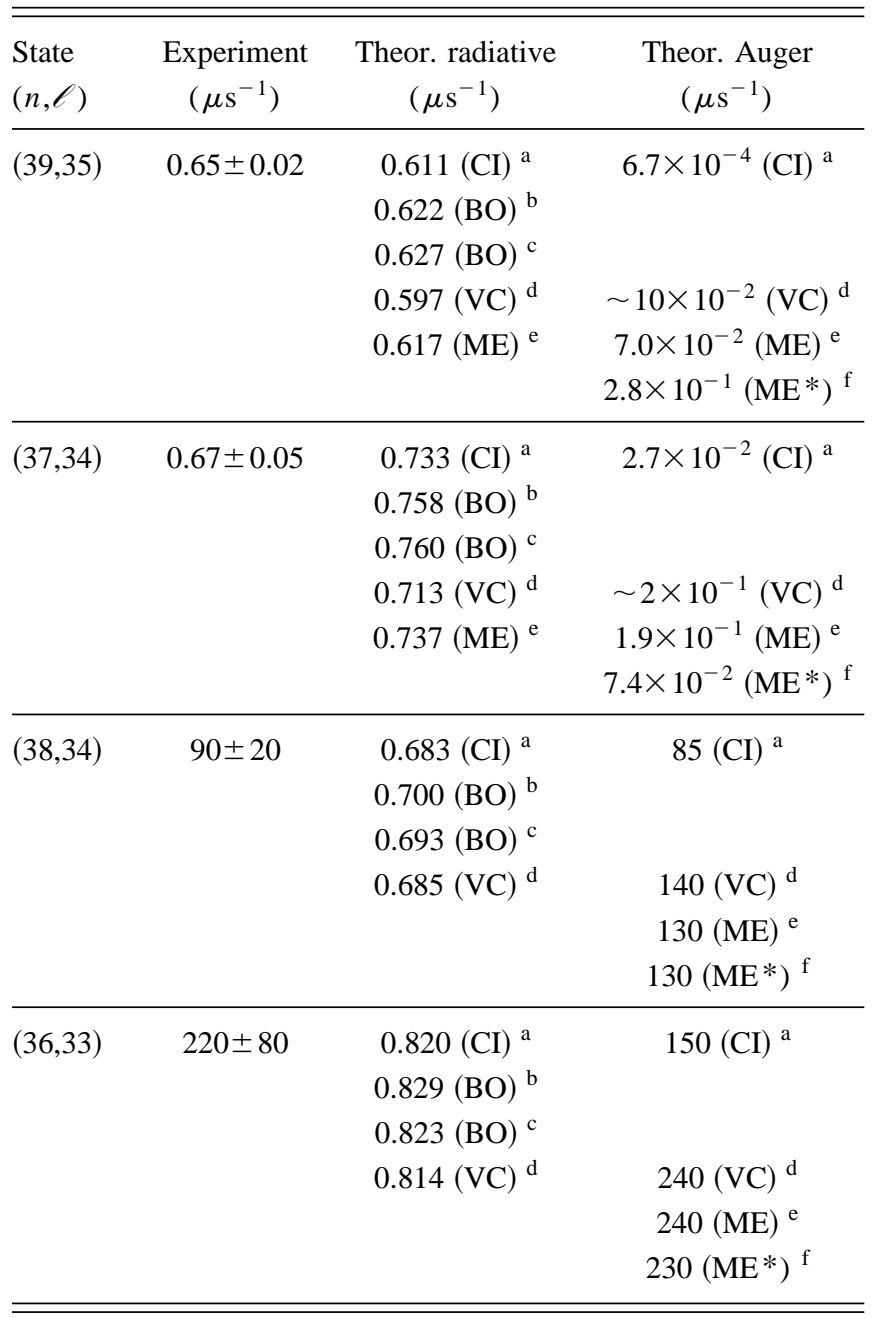

${ }^{\mathrm{a}}$ Configuration-interaction calculation from Ref. [24].

${ }^{b}$ Born-Oppenheimer calculation from Ref. [26].

${ }^{\mathrm{c}}$ Born-Oppenheimer calculation from Ref. [27].

${ }^{\mathrm{d}}$ Variational calculation from Refs. [30,31].

${ }^{\mathrm{e}}$ Molecular-expansion variational calculation from Refs. [33,34].

${ }^{\mathrm{f}}$ Auger calculation from Ref. [35], using molecular-expansion wave functions from Ref. [32].

$$
\begin{gathered}
R_{n, \ell}=\sum_{\Delta n} R_{n, \ell \rightarrow n+\Delta n, \ell \pm 1}, \\
A_{n, \ell}=\sum_{\Delta n, \Delta \ell} A_{n, \ell \rightarrow n+\Delta n, \ell+\Delta \ell} .
\end{gathered}
$$

Here $R_{n, \ell \rightarrow n+\Delta n, \ell \pm 1}$ is the radiative rate for individual transitions between states $(n, \mathscr{\ell})$ and $(n+\Delta n, \mathscr{\ell} \pm 1)$, whereas $A_{n, \ell \rightarrow n+\Delta n, \ell+\Delta \ell}$ is the internal Auger rate for transitions between states $(n, \mathscr{\ell})$ and $(n+\Delta n, \mathscr{\ell}+\Delta \mathscr{\ell})$ having a multiplicity $L=|\Delta \mathscr{\ell}|$. The summation is over all the possible decay channels. The term $D_{n, \ell}(\rho)$ represents the total rate of density-dependent processes depleting the state $(n, \mathscr{\ell})$, so that this effect disappears at zero density.
The wave functions, level energies, and state lifetimes of isolated $\bar{p} \mathrm{He}^{+}$atoms have recently been calculated to high precision in a number of theoretical approaches. In one of these the electronic motion is expressed using atomic Slatertype basis functions centered on the helium nucleus and the antiprotonic basis functions are calculated in the adiabatic potential of the $1 \mathrm{~s}_{\sigma}$ electronic state; the total atomic configuration-interaction wave functions are then obtained as a linear combination of products of these basis functions [25]. Molecular Born-Oppenheimer calculations have also been carried out, in which the $\bar{p} \mathrm{He}^{+}$system is considered as a one-electron molecule with two nuclei (the antiproton and the helium nucleus) and the electronic motion is separated adiabatically from the low-vibrational and high-rotational motions between the antiproton and the helium nucleus $[26,27]$. A variational calculation of the three-body system has also been done using a set of simple trial functions [30], while another molecular-expansion variational calculation used correlated trial functions that take into account the nonadiabatic electronic motion by including contributions from the azimuthal magnetic components $\sigma, \pi$, and $\delta$ of the electronic orbital [32]. Recently, the latter method has been expanded to include the relativistic motion of the electron [33].

\section{A. Radiative decay rates}

The radiative transition rates between two given states $\left(R_{n, \ell \rightarrow n+\Delta n, \ell \pm 1}\right)$ calculated using atomic configurationinteraction [24,25], molecular Born-Oppenheimer [26,27], and variational wave functions $[30,33]$ are in mutual agreement within $10 \%$. According to these calculations, the net radiative decay rates of the states $(n, \mathscr{\ell})=(39,35)$ and $(37,34)$ are around $0.6 \mu \mathrm{s}^{-1}$ and $0.7 \mu \mathrm{s}^{-1}$, respectively (see Table I). Due to the effect of the Coulomb polarization of the electronic cloud by the antiproton, the radiative rates are decreased by a factor of 3 compared to the results of calculations in which such electron-antiproton correlations are neglected.

In Fig. 12 the decay rates of the states $(39,35)$ and $(37,34)$ derived from the experiment are plotted as a function of density. When linearly extrapolated to zero density, the decay rate of state $(39,35)$ was $\lambda=0.65 \pm 0.02 \mu \mathrm{s}^{-1}$ (Fig. 12 , left), whereas the decay rate of state $(37,34)$ at zero density was $\lambda=0.67 \pm 0.05 \mu \mathrm{s}^{-1}$ when only the seven data points at low density (Fig. 12, right) are linearly extrapolated. These values agree with the calculated radiative rates within the experimental error.

\section{B. Internal Auger decay rates}

The Auger rates $\left(A_{n, \ell \rightarrow n+\Delta n, \ell+\Delta \ell}\right)$ have been calculated using the atomic configuration-interaction [24] and molecular variational [30,31,33-35] wave functions by applying a first-order perturbation formula (Fermi's golden rule), while a variational scattering calculation based on a molecular expansion of the wave functions has also been done [33,34]. The results indicate that internal Auger processes dominate $\left(A_{n, \ell \rightarrow n+\Delta n, \ell+\Delta \ell}>10 \mu \mathrm{s}^{-1}\right)$ for states from which multiplicity $L=|\Delta \mathscr{\ell}| \leqslant 3$ transitions to energetically lower-lying $\bar{p} \mathrm{He}^{2+}$ states are possible. The $L=3$ Auger transition rates 


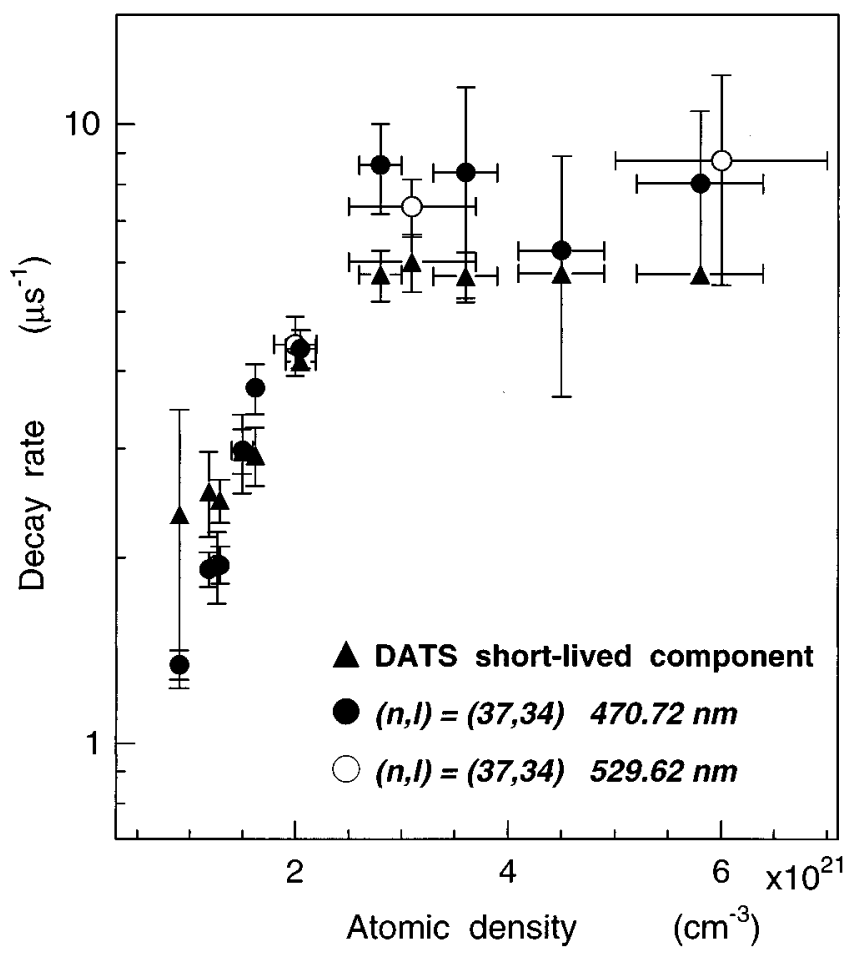

FIG. 12. Decay rates of the states $(n, \ell)=(39,35)$ and $(37,34)$ measured at various densities using the 597.26-nm, 470.72-nm, and 529.62-nm resonances. Radiative rates from Born-Oppenheimer (BO [26,27]) and variational calculations $\left(\mathrm{VC}_{\mathrm{rad}}[30]\right.$ and $\mathrm{ME}_{\mathrm{rad}}$ [33]) and the sum of Auger and radiative rates from atomic configuration-interaction (CI [24]) and variational calculations $\left(\mathrm{VC}_{\mathrm{Aug}}[30,31], \mathrm{ME}_{\mathrm{Aug}}\right.$ [34], and $\mathrm{ME}_{\text {Aug }}^{*}$ [35]) are shown.

calculated by these methods agree within a factor of 2 (see Table I); the precision of the molecular variational calculations is claimed to be $10 \%$ according to Refs. [30,31,33]. In contrast, the calculations of the strongly suppressed $L \geqslant 4$ Auger transition rates are complicated by the small values of the transition matrix element, so that only rough estimates are presently available [24,30,31,33].

For the Auger-dominated states $(n, \ell)=(38,34)$ and $(36,33)$, the measured decay rates $90 \pm 20 \mu \mathrm{s}^{-1}$ and 220 $\pm 80 \mu \mathrm{s}^{-1}$, respectively, agree with the results of calculations within experimental error (see Table I). For the metastable states $(39,35)$ and $(37,34)$, upper limits of the Auger rates can be obtained by comparing the calculated radiative rates with the experimentally measured zero-density decay rates (Figs. 8 and 12). The upper limits for the Auger rates of both states are $\sim 0.1 \mu \mathrm{s}^{-1}$, which agrees with the decay rates calculated by atomic configuration-interaction and molecular variational methods.

\section{Density-dependent lifetime shortening}

It appears that collisions with external helium atoms strongly reduce the lifetimes of the lower-lying metastable state $(n, \mathscr{\ell})=(37,34)$, while they leave the energetically higher-lying state $(39,35)$ unaffected. The state $(39,35)$ retained the radiative rate of $0.7 \mu \mathrm{s}^{-1}$ even at liquid-helium densities, whereas the decay rate of the state $(37,34)$ increased nonlinearly with target density, from the purely radiative value of $\lambda=0.8 \mu \mathrm{s}^{-1}$ at $\rho=10^{20} \mathrm{~cm}^{-3}$ to $\lambda \sim 8 \mu \mathrm{s}^{-1}$

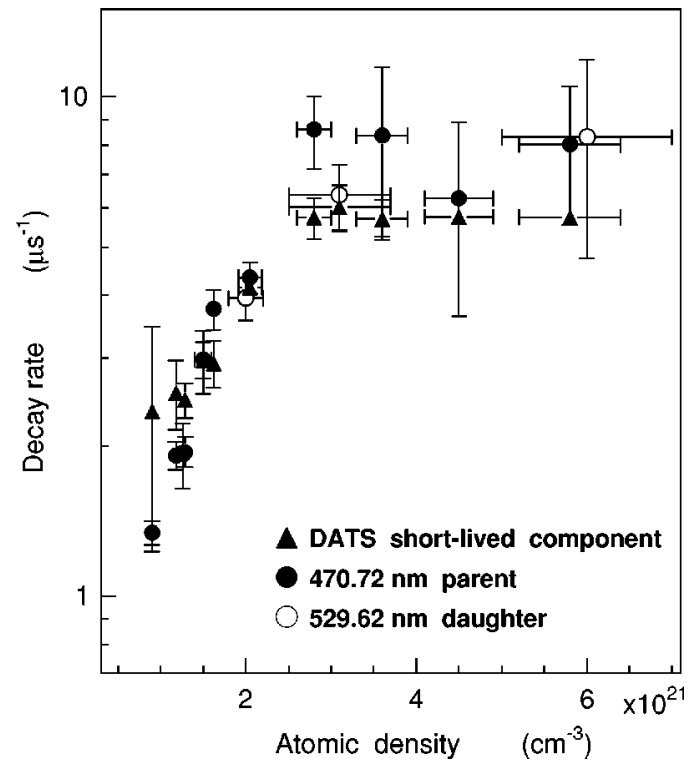

FIG. 13. Correlation between the decay rates of the state $(n, \ell)=(37,34)$ measured using the 470.72-nm and 529.62-nm resonances, and the short-lived component in the delayed annihilation time spectrum (DATS). The decay rates are in good agreement at densities between $10^{21}$ and $6 \times 10^{21} \mathrm{~cm}^{-3}$, indicating that the state $(37,34)$ is one of the principal contributors to the short-lived component.

at $\rho=3 \times 10^{21} \mathrm{~cm}^{-3}$, then leveled off at higher densities (see Fig. 12). At present, we have no understanding of the mechanisms that cause this state-dependent lifetime shortening.

The delayed annihilation time spectrum was found to contain several decay components that become short lived in high-density helium, while others remain relatively unaffected. The time spectrum is essentially a summation of antiprotons cascading through all possible channels before annihilation, so that several conclusions can be drawn from it concerning the reaction of the $\bar{p} \mathrm{He}^{+}$atom to collisions. First, the lifetimes of some levels are greatly shortened with increasing density, while those of others are not. One or more density-shortened state(s) must exist to account for the $\lambda \sim 6 \mu \mathrm{s}^{-1}$ short-lived component in the delayed annihilation time spectrum at densities above $3 \times 10^{21} \mathrm{~cm}^{-3}$. The majority of metastable levels, however, must retain their radiative lifetimes, since the long-lived component containing most of the decaying $\bar{p} \mathrm{He}^{+}$changes relatively little $(\lambda$ $\sim 0.15 \mu \mathrm{s}^{-1}$ at $\rho=2 \times 10^{20} \mathrm{~cm}^{-3}, \lambda \sim 0.4 \mu \mathrm{s}^{-1}$ in liquid helium at $\left.\rho=2 \times 10^{22} \mathrm{~cm}^{-3}[1-4]\right)$.

Second, in order to account for the short-lived component, direct antiproton capture into at least one of these densitysensitive states must occur when the $\bar{p} \mathrm{He}^{+}$atom is initially formed. If the antiproton were instead captured in higherlying metastable states, the time required for cascading down into the density-sensitive state would introduce an additional delay and never produce such a short-lived component.

These conclusions are proved by the present laser spectroscopic study of individual state lifetimes, in which we have identified a specific density shortened state $(37,34)$ that lies at the border between metastable and Auger-dominated states in the initially populated region $n \approx \sqrt{M^{*} / m_{e}}=38.3$. The lifetime of this state is in good agreement with the life- 
TABLE II. Decay rates of the radiation-dominated states $(n, \mathscr{\ell})=(39,35)$ and $(37,34)$, measured at various target densities, and decay rates of the delayed annihilation time spectrum (DATS). The time region $t=0.1-3 \mu \mathrm{s}$ following $\bar{p} \mathrm{He}^{+}$atom formation is fitted with two components $\lambda_{1}$ and $\lambda_{2}$.

\begin{tabular}{|c|c|c|c|c|c|}
\hline $\begin{array}{c}\text { Density } \\
\left(10^{20} \mathrm{~cm}^{-3}\right)\end{array}$ & $\begin{array}{l}\text { Pressure } \\
\text { (bar) }\end{array}$ & $\begin{array}{c}\text { Temperature } \\
(\mathrm{K})\end{array}$ & $\begin{array}{l}\text { State decay rate } \\
\qquad\left(\mu \mathrm{s}^{-1}\right)\end{array}$ & $\begin{array}{c}\operatorname{DATS} \lambda_{1} \\
\left(\mu \mathrm{s}^{-1}\right)\end{array}$ & $\begin{array}{c}\text { DATS } \lambda_{2} \\
\left(\mu \mathrm{s}^{-1}\right)\end{array}$ \\
\hline \multicolumn{6}{|c|}{$(n, \mathscr{C})=(39,35)$ state using the 597.26-nm resonance } \\
\hline $2.0 \pm 0.3$ & $0.19 \pm 0.02$ & $6.8 \pm 0.3$ & $0.66 \pm 0.06$ & $3 \pm 2$ & $0.15 \pm 0.01$ \\
\hline $6.6 \pm 0.2$ & $0.55 \pm 0.02$ & $6.3 \pm 0.1$ & $0.69 \pm 0.05$ & $17.1 \pm 0.9$ & $0.199 \pm 0.003$ \\
\hline $7.1 \pm 0.3$ & $0.59 \pm 0.02$ & $6.4 \pm 0.2$ & $0.64 \pm 0.03$ & $7 \pm 2$ & $0.204 \pm 0.002$ \\
\hline $12.5 \pm 0.5$ & $1.00 \pm 0.03$ & $6.4 \pm 0.2$ & $0.63 \pm 0.02$ & $2.53 \pm 0.05$ & $0.250 \pm 0.003$ \\
\hline $150 \pm 10$ & $4.5 \pm 0.2$ & $5.8 \pm 0.1$ & $0.69 \pm 0.07$ & $7.0 \pm 0.1$ & $0.377 \pm 0.006$ \\
\hline $194 \pm 7$ & $8.7 \pm 0.3$ & $5.8 \pm 0.1$ & $0.68 \pm 0.07$ & $7.0 \pm 0.2$ & $0.419 \pm 0.009$ \\
\hline \multicolumn{6}{|c|}{$(n, \mathscr{\ell})=(37,34), 470.72 \mathrm{~nm}$} \\
\hline $1.2 \pm 0.1$ & $0.100 \pm 0.007$ & $6.3 \pm 0.2$ & $0.8 \pm 0.1$ & $1.1 \pm 0.1$ & $0.133 \pm 0.003$ \\
\hline $1.5 \pm 0.1$ & $0.121 \pm 0.007$ & $6.0 \pm 0.2$ & $0.8 \pm 0.2$ & $2.4 \pm 0.5$ & $0.122 \pm 0.006$ \\
\hline $2.0 \pm 0.2$ & $0.17 \pm 0.01$ & $6.3 \pm 0.2$ & $0.84 \pm 0.08$ & $2.5 \pm 0.4$ & $0.147 \pm 0.003$ \\
\hline $2.5 \pm 0.2$ & $0.22 \pm 0.01$ & $6.4 \pm 0.2$ & $0.86 \pm 0.03$ & $4.0 \pm 0.1$ & $0.137 \pm 0.003$ \\
\hline $4.4 \pm 0.5$ & $0.37 \pm 0.03$ & $6.3 \pm 0.2$ & $0.99 \pm 0.07$ & $26 \pm 7$ & $0.157 \pm 0.002$ \\
\hline $6.9 \pm 0.3$ & $0.57 \pm 0.01$ & $6.4 \pm 0.2$ & $1.25 \pm 0.07$ & $17.0 \pm 0.9$ & $0.206 \pm 0.002$ \\
\hline $9.0 \pm 0.3$ & $0.729 \pm 0.06$ & $6.3 \pm 0.1$ & $1.34 \pm 0.07$ & $2 \pm 1$ & $0.223 \pm 0.004$ \\
\hline $11.8 \pm 0.3$ & $0.94 \pm 0.03$ & $6.3 \pm 0.1$ & $1.9 \pm 0.1$ & $2.6 \pm 0.4$ & $0.25 \pm 0.01$ \\
\hline $12.9 \pm 0.4$ & $1.07 \pm 0.02$ & $6.6 \pm 0.2$ & $1.9 \pm 0.1$ & $2.5 \pm 0.2$ & $0.25 \pm 0.01$ \\
\hline $15 \pm 1$ & $1.13 \pm 0.08$ & $6.3 \pm 0.1$ & $3.0 \pm 0.3$ & $3.0 \pm 0.4$ & $0.27 \pm 0.01$ \\
\hline $16.2 \pm 0.7$ & $1.05 \pm 0.04$ & $5.5 \pm 0.2$ & $3.8 \pm 0.3$ & $3.0 \pm 0.3$ & $0.26 \pm 0.02$ \\
\hline $20 \pm 1$ & $1.29 \pm 0.07$ & $5.5 \pm 0.2$ & $4.4 \pm 0.3$ & $4.1 \pm 0.1$ & $0.294 \pm 0.005$ \\
\hline $28 \pm 2$ & $1.73 \pm 0.08$ & $5.8 \pm 0.1$ & $9 \pm 1$ & $5.7 \pm 0.5$ & $0.33 \pm 0.01$ \\
\hline $36 \pm 3$ & $2.1 \pm 0.1$ & $5.8 \pm 0.2$ & $8 \pm 3$ & $5.7 \pm 0.5$ & $0.36 \pm 0.01$ \\
\hline $45 \pm 4$ & $2.4 \pm 0.1$ & $5.8 \pm 0.2$ & $6 \pm 3$ & $5.8 \pm 0.1$ & $0.384 \pm 0.005$ \\
\hline $58 \pm 6$ & $2.72 \pm 0.06$ & $5.8 \pm 0.1$ & $8 \pm 2$ & $5.7 \pm 0.1$ & $0.389 \pm 0.006$ \\
\hline \multicolumn{6}{|c|}{$(n, \mathscr{\ell})=(37,34), 529.62 \mathrm{~nm}$} \\
\hline $12.6 \pm 0.5$ & $1.03 \pm 0.04$ & $6.5 \pm 0.2$ & $1.9 \pm 0.3$ & $2.4 \pm 0.4$ & $0.24 \pm 0.01$ \\
\hline $20 \pm 2$ & $1.19 \pm 0.07$ & $5.3 \pm 0.2$ & $4.4 \pm 0.5$ & $4.04 \pm 0.07$ & $0.287 \pm 0.004$ \\
\hline $31 \pm 6$ & $1.9 \pm 0.1$ & $5.8 \pm 0.1$ & $7.7 \pm 0.9$ & $6.0 \pm 0.6$ & $0.34 \pm 0.01$ \\
\hline $60 \pm 10$ & $2.7 \pm 0.3$ & $5.8 \pm 0.2$ & $8 \pm 2$ & $5.6 \pm 0.2$ & $0.39 \pm 0.01$ \\
\hline
\end{tabular}

time of the short-lived component that appears at early time regions in the delayed annihilation time spectrum at densities between $10^{21}$ and $6 \times 10^{21} \mathrm{~cm}^{-3}$ (see Fig. 13 and Table II). This indicates that the state $(37,34)$ is one of the principal contributors to this short-lived component. An example of a state not affected by density changes was also found: $(39,35)$, which is presumed to contribute to the long-lived component in the delayed annihilation time spectrum that is relatively unaffected by density.

\section{SUMMARY AND CONCLUSIONS}

The three resonances of metastable antiprotonic helium $\left(\bar{p} \mathrm{He}^{+}\right)$atoms, $(n, \mathscr{\ell})=(39,35) \rightarrow(38,34)$ at $597.26 \mathrm{~nm}$, $(37,34) \rightarrow(36,33)$ at $470.72 \mathrm{~nm}$, and $(38,35) \rightarrow(37,34)$ at $529.62 \mathrm{~nm}$, were studied using laser-induced annihilation, for $\bar{p} \mathrm{He}^{+}$atoms formed in pure helium gas at pressures between 0.1 and 9.0 bars, and temperatures between 5.5 and 7.0 K. A laser spectroscopic method was developed to directly measure the lifetimes of individual states, and the lifetimes of the two metastable states $(39,35)$ and $(37,34)$ together with two states with large Auger widths $(38,34)$ and
$(36,33)$ were obtained. The time spectra of these resonances show depletion-recovery profiles, characterized by a decay with the lifetimes of the resonance daughter states, and a recovery with the lifetimes of the parent states. Using these characteristics, the lifetimes of the parent and daughter states can be directly determined in a model-independent way. The state $(39,35)$ had a zero-density decay rate of $0.65 \mu \mathrm{s}^{-1}$, with no significant change between densities of $2 \times 10^{20}$ and $1.9 \times 10^{22} \mathrm{~cm}^{-3}$. In sharp contrast, the decay rate of the state $(37,34)$ increased nonlinearly with target density, from 0.8 $\mu \mathrm{s}^{-1}$ at $1.2 \times 10^{20} \mathrm{~cm}^{-3}$ to $\sim 8 \mu \mathrm{s}^{-1}$ at $5.8 \times 10^{21} \mathrm{~cm}^{-3}$. This state is responsible for the short-lived component that appears in the overall delayed annihilation time spectra at densities above $10^{21} \mathrm{~cm}^{-3}$. Using the shortened lifetime of the state at high density, the resonance $(38,35) \rightarrow(37,34)$ at $529.62 \mathrm{~nm}$ was detected. The measured lifetimes of the metastable states $(39,35)$ and $(37,34)$ at the zero density limit agreed with radiative rates calculated with configuration-interaction, Born-Oppenheimer, and molecular variational methods. The Auger-dominated states $(38,34)$ and $(36,33)$ had decay rates of $90 \pm 20 \mu \mathrm{s}^{-1}$ and $220 \pm 80$ $\mu \mathrm{s}^{-1}$, respectively, which agree with Auger rates calculated 
using configuration-interaction and molecular variational wave-functions.

\section{ACKNOWLEDGMENTS}

We appreciate the hospitality and support provided by CERN staff during our experiment. We would particularly like to thank the PS division staff for their untiring efforts in providing us with a stable antiproton beam. We are indebted to the CERN cryogenics laboratory for their expertise in de- signing, manufacturing, and testing our target cryostat. We wish to thank V. I. Korobov, K. Ohtsuki, and O. I. Kartavtsev for many valuable discussions. The present work was supported by the Grants-in-Aid for Specially Promoted Research and for International Scientific Research of the Japanese Ministry of Education, Science, Sports and Culture, the German Bundesministerium für Bildung, Wissenschaft, Forschung und Technologie, and the Hungarian National Science Foundation. H. A. T. acknowledges support from the Japanese Society for Promotion of Science.
[1] M. Iwasaki, S. N. Nakamura, K. Shigaki, Y. Shimizu, H. Tamura, T. Ishikawa, R. S. Hayano, E. Takada, E. Widmann, H. Outa, M. Aoki, P. Kitching, and T. Yamazaki, Phys. Rev. Lett. 67, 1246 (1991).

[2] T. Yamazaki, E. Widmann, R. S. Hayano, M. Iwasaki, S. N. Nakamura, K. Shigaki, F. J. Hartmann, H. Daniel, T. von Egidy, P. Hofmann, Y. -S. Kim, and J. Eades, Nature (London) 361, 238 (1993).

[3] S. N. Nakamura, R. S. Hayano, M. Iwasaki, K. Shigaki, E. Widmann, T. Yamazaki, H. Daniel, T. von Egidy, F. J. Hartmann, P. Hofmann, Y.-S. Kim, and J. Eades, Phys. Rev. A 49, 4457 (1994).

[4] E. Widmann, I. Sugai, T. Yamazaki, R. S. Hayano, M. Iwasaki, S. N. Nakamura, H. Tamura, T. M. Ito, N. Morita, F. J. Hartmann, H. Daniel, T. von Egidy, W. Schmid, J. Hoffmann, and J. Eades, Phys. Rev. A 51, 2870 (1995).

[5] B. Ketzer, F. J. Hartmann, H. Daniel, T. von Egidy, A. Niestroj, S. Schmid, W. Schmid, T. Yamazaki, I. Sugai, K. Nakayoshi, R. S. Hayano, F. E. Maas, H. A. Torii, T. Ishikawa, H. Tamura, N. Morita, D. Horváth, J. Eades, and E. Widmann, Phys. Rev. A 53, 2108 (1996).

[6] E. Widmann, I. Sugai, T. Yamazaki, R. S. Hayano, M. Iwasaki, S. N. Nakamura, H. Tamura, T. M. Ito, A. Kawachi, N. Nishida, W. Higemoto, Y. Ito, N. Morita, F. J. Hartmann, H. Daniel, T. von Egidy, W. Schmid, J. Hoffmann, and J. Eades, Phys. Rev. A 53, 3129 (1996).

[7] G. T. Condo, Phys. Lett. 9, 65 (1964).

[8] J. E. Russell, Phys. Rev. Lett. 23, 63 (1969); Phys. Rev. 188, 187 (1969); Phys. Rev. A 1, 721 (1970); 1, 735 (1970); 1, 742 (1970); J. Math. Phys. 12, 1906 (1971); Phys. Rev. A 6, 2488 (1972).

[9] N. Morita, K. Ohtsuki, and T. Yamazaki, Nucl. Instrum. Methods Phys. Res. A 330, 439 (1993).

[10] N. Morita, M. Kumakura, T. Yamazaki, E. Widmann, H. Masuda, I. Sugai, R. S. Hayano, F. E. Maas, H. A. Torii, F. J. Hartmann, H. Daniel, T. von Egidy, B. Ketzer, W. Müller, W. Schmid, D. Horváth, and J. Eades, Phys. Rev. Lett. 72, 1180 (1994).

[11] R. S. Hayano, F. E. Maas, H. A. Torii, N. Morita, M. Kumakura, T. Yamazaki, H. Masuda, I. Sugai, F. J. Hartmann, H. Daniel, T. von Egidy, B. Ketzer, W. Müller, W. Schmid, D. Horváth, J. Eades, and E. Widmann, Phys. Rev. Lett. 73, 1485 (1994); 73, 3181(E) (1994).

[12] F. E. Maas, R. S. Hayano, T. Ishikawa, H. Tamura, H. A. Torii, N. Morita, T. Yamazaki, I. Sugai, K. Nakayoshi, F. J. Hartmann, H. Daniel, T. von Egidy, B. Ketzer, A. Niestroj, S.
Schmid, W. Schmid, D. Horváth, J. Eades, and E. Widmann, Phys. Rev. A 52, 4266 (1995).

[13] H. A. Torii, M. Hori, T. Ishikawa, F. E. Maas, R. S. Hayano, N. Morita, M. Kumakura, I. Sugai, B. Ketzer, H. Daniel, F. J. Hartmann, R. Pohl, R. Schmidt, T. von Egidy, D. Horváth, J. Eades, E. Widmann, and T. Yamazaki, Phys. Rev. A 53, R1931 (1996).

[14] R. S. Hayano, T. Ishikawa, H. Tamura, H. A. Torii, M. Hori, F. E. Maas, N. Morita, M. Kumakura, I. Sugai, F. J. Hartmann, H. Daniel, T. von Egidy, B. Ketzer, R. Pohl, D. Horváth, J. Eades, E. Widmann, and T. Yamazaki, Phys. Rev. A 55, R1 (1997).

[15] T. Yamazaki, B. Ketzer, E. Widmann, J. Eades, H. Daniel, F. J. Hartmann, M. Hasinoff, R. Pohl, R. Schmidt, T. von Egidy, D. Horváth, M. Kumakura, N. Morita, I. Sugai, Y. Fujita, H. A. Torii, M. Hori, T. Ishikawa, F. E. Maas, H. Tamura, and R. S. Hayano, Chem. Phys. Lett. 265, 137 (1997).

[16] B. Ketzer, F. J. Hartmann, T. von Egidy, C. Maierl, R. Pohl, J. Eades, E. Widmann, T. Yamazaki, M. Kumakura, N. Morita, R. S. Hayano, M. Hori, T. Ishikawa, H. A. Torii, I. Sugai, and D. Horváth, Phys. Rev. Lett. 78, 1671 (1997).

[17] T. Yamazaki, E. Widmann, J. Eades, M. Kumakura, N. Morita, H. A. Torii, M. Hori, T. Ishikawa, F. E. Maas, H. Tamura, R. S. Hayano, I. Sugai, Y. Fujita, H. Daniel, B. Ketzer, H. Daniel, F. J. Hartmann, R. Pohl, R. Schmidt, T. von Egidy, and D. Horváth, Phys. Rev. A 55, R3295 (1997).

[18] E. Widmann, J. Eades, T. Yamazaki, H. A. Torii, R. S. Hayano, M. Hori, T. Ishikawa, M. Kumakura, N. Morita, I. Sugai, F. J. Hartmann, T. von Egidy, B. Ketzer, C. Maierl, R. Pohl, and D. Horváth, Phys. Lett. B 404, 15 (1997).

[19] H. A. Torii, R. S. Hayano, F. E. Maas, N. Morita, M. Kumakura, T. Yamazaki, H. Masuda, I. Sugai, B. Ketzer, F. J. Hartmann, H. Daniel, T. von Egidy, W. Müller, W. Schmid, D. Horváth, J. Eades, and E. Widmann, Nucl. Instrum. Methods Phys. Res. A 396, 257 (1997).

[20] T. B. Day, G. A. Snow, and J. Sucher, Phys. Rev. Lett. 3, 61 (1959).

[21] M. Leon and H. A. Bethe, Phys. Rev. 127, 636 (1962).

[22] A review is given in F. J. Hartmann, Electromagnetic Cascade and Chemistry of Exotic Atoms (Plenum, New York, 1990), pp. 127-139.

[23] R. Ahlrichs, O. Dumbrajs, H. Pilkuhn, and H. G. Schlaile, Z. Phys. A 306, 297 (1982).

[24] K. Ohtsuki (private communication).

[25] T. Yamazaki and K. Ohtsuki, Phys. Rev. A 45, 7782 (1992).

[26] I. Shimamura, Phys. Rev. A 46, 3776 (1992); and private communication. 
[27] P. T. Greenland and R. Thürwächter, Hyperfine Interact. 76, 355 (1993), and private communication.

[28] T. B. Day, Nuovo Cimento 18, 381 (1960).

[29] G. Reifenröther, E. Klempt, and R. Landua, Phys. Lett. B 203, 9 (1988).

[30] O. I. Kartavtsev, At. Nucl. 59, 1483 (1996).

[31] S. I. Fedotov, O. I. Kartavtsev, and D. E. Monakhov, At. Nucl. 59, 1662 (1996).

[32] V. I. Korobov, Phys. Rev. A 54, R1749 (1996).

[33] V. I. Korobov, Nucl. Phys. B 56A, 89 (1997), and private communication.

[34] V. I. Korobov and I. Shimamura, Phys. Rev. A 56, 4587 (1997).

[35] J. Révai and A. T. Kruppa, Phys. Rev. A 57, 174 (1998), and private communication.

[36] Cryodata Inc., HELIUM THERMOPHYSICAL PROPERTIES SOFTWARE HEPAK, version 3.30, 1992.

[37] M. Durieux and R. K. Rusby, Metrologia 19, 67 (1983).

[38] M. Iwasaki, LOW ENERGY PARTICLE SIMULATOR, version 6.6, 1996, a Monte Carlo simulation to estimate the energy loss of low-energy charged particles in various materials, including the multiple scattering effect according to the Moliere distribution, the energy straggling effect according to the Vavilov distribution, and the Barkas effect, based on J. F. Ziegler, The Stopping and Range of Ions in Solid (Pergamon, New York, 1985).

[39] J.-Y. Hémery and D. J. Simon, CERN Report No. PS/9228(PA), 1992 (unpublished). 